Alberto José Figueras *

Daniela Cristina **

Valeria Blanco ****

Iván Iturralde $* * * * * *$

Marcelo Luis Capello *****\%*

Universidad Nacional de Córdoba, Córdoba, Argentina.

Recibido: 2 de abril de 2014

Concepto de evaluación: 11 de junio de 2014

Aprobado: 18 de junio de 2014

Artículo de investigación

(C) 2014 Universidad Católica de

Colombia. Facultad de Ciencias

Económicas y Administrativas.

Todos los derechos reservados

* Doctor en Economía. Vicedirector del Instituto de Economía y Finanzas y profesor titular de la Universidad Nacional de Córdoba. Dirección de correspondencia: Av. Valparaíso s/n Ciudad Universitaria. Facultad de Ciencias Económicas, Universidad Nacional de Córdoba (Argentina). Correo electrónico: alfi@eco.unc.edu.ar

** Doctora en Economía. Profesora asistente, Universidad Nacional de Córdoba. Dirección de correspondencia: Av. Valparaíso $s / n$ Ciudad Universitaria. Facultad de Ciencias Económicas,

Universidad Nacional de Córdoba (Argentina). Correo electrónico: daniela.cristina@eco.uncor.edu

*** Magíster en Economía. Profesora ayudante, Universidad Nacional de Córdoba. Dirección de correspondencia: Av. Valparaíso $\mathrm{s} / \mathrm{n}$ Ciudad Universitaria. Facultad de Ciencias Económicas, Universidad Nacional de Córdoba (Argentina). Correo electrónico: valeriablanco@eco.unc.edu.ar
Finanz. polit. econ., ISSN: 2248-6046, Vol. 6, No. 2, julio-diciembre, 2014, pp. 287-316

http://dx.doi.org/10.14718/revfinanzpolitecon.2014.6.2.4

\section{Un aporte al debate sobre la convergencia en Argentina: la importancia de los cambios estructurales $^{1}$}

\section{RESUMEN}

El análisis del crecimiento económico de las provincias argentinas, signado por su comportamiento idiosincrático, es un valioso aporte a los estudios de convergencia regional. En el presente trabajo, se propone un análisis de Convergencia Beta y Sigma de las provincias argentinas para el periodo 1970-2007, con variables que aproximan el capital humano, las economías de escala, la inversión y la política fiscal. Adicionalmente, se intenta aislar el efecto de shocks sobre el crecimiento económico de provincias con diferentes estructuras productivas, y sus consecuencias sobre la convergencia/divergencia regional en Argentina.

Palabras clave: crecimiento, convergencia, economías regionales.

JEL: O4, R0, E6

\section{A Contribution to the Discussion about Convergence in Argentina: the Importance of Structural Changes}

\section{ABSTRACT}

Since Argentinian provinces' economic growth is determined by idiosyncratic behaviour, its analysis is a valuable contribution to the studies on regional convergence. This paper takes on an analysis of Sigma and Beta convergence in Argentinian provinces for the 1970-2007 period, with variables that include human capital, economies of scale, investment and fiscal policy. It also focuses on the effect structural changes exert upon provinces'

1 Existe una versión anterior del tema, expuesta en la reunión de la Asociación Argentina de Economía Política (AAEP): Estudio sobre el crecimiento económico: un análisis de convergencia, con autoría de Figueras, Cristina, Blanco y Capello (2013). 
**** Licenciado en Economía y candidato a doctor en

Economía. Profesor ayudante, Universidad Nacional de Córdoba. Dirección de correspondencia: Av. Valparaíso s/n Ciudad Universitaria. Facultad de Ciencias Económicas, Universidad Nacional de Córdoba

(Argentina). Correo electrónico: ivit@eco.unc.edu.ar

$* * * * *$ Magíster en Economía. Profesor adjunto, Universidad Nacional de Córdoba, presidente del IERAL de Fundación Mediterránea.

Dirección de correspondencia: Av. Valparaíso s/n Ciudad Universitaria. Facultad de Ciencias Económicas, Universidad Nacional de Córdoba (Argentina). Correo electrónico: mcapello@eco.unc.edu.ar economic growth -with their different productive structures- and its impact on the regional convergence/divergence in Argentina.

Keywords: Growth, convergence, regional economies.

\section{Uma contribuição para o debate sobre a convergência na Argentina: a importância das mudanças estruturais}

\section{RESUMO}

A análise do crescimento econômico das províncias argentinas, marcado por seu comportamento idiossincrático, é uma valiosa contribuição para os estudos de convergência regional. No presente trabalho, propõe-se uma análise de convergência beta e sigma das províncias argentinas para o período de 1970-2007, com variáveis que aproximam o capital humano, as economias de escala, o investimento e a política fiscal. Além disso, tenta-se isolar o efeito de choques sobre o crescimento econômico de províncias com diferentes estruturas produtivas e suas consequências sobre a convergência/divergência regional na Argentina.

Palavras-chave: crescimento, convergência, economias regionais. 


\section{INTRODUCCIÓN}

La preocupación por el crecimiento y su potenciación ha sido el norte de las reflexiones de la economía desde sus inicios. Pese a los numerosos estudios con diversas variables, a lo largo y ancho del mundo, es válido trabajar el caso para las provincias argentinas; si hay un país con conductas idiosincrásicas, ese país es Argentina, y es muy posible que lo válido en otras latitudes no lo sea aquí.

No hay que olvidar que el mundo vive un periodo, desde hace dos décadas (y pese a la crisis iniciada en el 2008) de auge, sustentado en la ola tecnológica (y la incorporación de China al proceso económico mundial); pero este periodo ha sido vivido por las distintas regiones con acentuadas diferencias de partida en sus dotaciones de factores (servicios sanitarios, nivel educativo, infraestructura física, capital humano, capital físico e, incluso, infraestructura social o institucional) y en su estructura productiva; lo que ha conducido a comportamientos muy diferenciados, y a una evolución conjunta que puede ser tanto convergente como divergente. De allí que se justifica, entendemos, encarar nuevamente el asunto empíricamente.

Dichas estas breves palabras a modo de introducción, lo que resta del presente trabajo se organiza así: en la primera parte, se realizan algunas consideraciones generales; en la segunda parte, se efectúan una serie de reflexiones teóricas sobre la convergencia social y la convergencia económica; en la tercera parte, se realizan algunas consideraciones sobre la conformación espacial de Argentina, haciendo unas breves referencias a los estudios anteriores sobre la misma temática en el espacio geográfico argentino; en la cuarta parte, se inicia la presentación de los aspectos que conforman el estudio empírico; la quinta parte presenta los resultados obtenidos en cuanto a la Convergencia Beta; mientras que en la sexta parte, se trata la Convergencia Sigma. Por último, se finaliza con un conjunto de reflexiones de cierre y un breve acápite metodológico.

\section{UNA PERSPECTIVA VIGENTE: LA CONVERGENCIA ECONÓMICA}

Como un derivado específico de la vieja tesis de la convergencia social, se plantea la convergencia en el crecimiento. Efectivamente, un tema de moda desde hace un cuarto de siglo, y cada vez más debatido en su vertiente teórica y empírica, es el llamado fenómeno de la convergencia económica. Se puede demostrar, en una decena de pasos matemáticos, que en una economía con un proceso de crecimiento convergente, posible de reproducir por un esquema teórico de tipo neoclásico, la tasa de crecimiento de la producción real per cápita (ingreso per cápita), en un periodo dado, está negativamente relacionada con el logaritmo del nivel de ingreso per cápita inicial, en ese periodo considerado. Cuanto más alto sea el ingreso por habitante de un país, menor será su tasa de crecimiento. Lo cual da lugar a la idea de convergencia entre los países ricos y los pobres (en ingreso per cápita), pues los primeros crecerán a tasas menores que los segundos.

Los modelos sugerían una relación inversa entre la tasa de crecimiento del ingreso y el nivel inicial del periodo (en especial, si las economías eran similares en preferencias y en tecnología); lo que promovería una aproximación en el nivel de ingreso entre pobres y ricos, donde los primeros alcanzarían a los segundos; convergiendo así teóricamente al mismo nivel de ingreso (o producto) por persona.

A mediados de los años ochenta, Baumol (1986) examinó el problema para dieciséis países industrializados, a lo largo del periodo centenario que va desde 1870 a 1979; para ello, utilizó la base de datos de Maddison (1982). Su conclusión fue que las cifras reflejaban una fuerte convergencia en el crecimiento de las naciones industriales desde 1870. Conceptualmente, esta captura (catch-up) de los ricos por los pobres estaría fundada en una difusión sin costo de las innovaciones tecnológicas. Esta contribución de Baumol ha abierto un amplio debate y ha originado diversos trabajos que se han ido multiplicando exponencialmente. Algunos respaldan su posición, en el sentido de 
que la convergencia ha operado vigorosamente entre las naciones industriales desde 1870. Otros, como el comentario de De Long (1988) sobre el artículo de Baumol, señalan problemas de sesgo en los datos y errores de medición en las variables, y sugieren que aquel estudio adolece de defectos y que, en realidad, no ha existido convergencia.

Este tipo de análisis no surgen de una preocupación ética del mundo desarrollado, sino más bien de un temor. En Estados Unidos existía, en los ochenta, la recelosa preocupación por la posibilidad de que su predominio mundial se viera desafiado ante la posibilidad de que la productividad del país fuera sobrepasada por otras naciones (v. gr., Japón o Alemania); lo que implicaría, a largo plazo, el deterioro de su hegemonía económica y militar.

El mismo Baumol (Baumol et al., 1989) fue quien introdujo el concepto de club de convergencia, a partir del cual afinaba la idea de 1986 y sostenía que Estados Unidos no sería sobrepasado, sino que sencillamente otros veinte países selectos lo irían alcanzando en un proceso de convergencia hacia un nivel de productividad similar. Técnicamente, se supone que estos países poseen un idéntico estado estacionario. En esta nueva obra, Baumol señala que esa aproximación no se da entre países cualesquiera, sino entre aquellos que están condicionados por similares circunstancias. Esto es, forman parte del mismo grupo o club. Emerge así la idea de una doble alternativa de modelar la convergencia: una convergencia absoluta (que operaría en el caso de idénticos estados estacionarios) y una condicional (posible en presencia de diferentes estados estacionarios).

Entonces, lo que realmente afirma el modelo neoclásico no es la convergencia entre distintas unidades macro, sino una aproximación al nivel de ingreso per cápita de equilibrio de largo plazo de cada economía; lo cual, en caso de existir equilibrios de largo plazo similares para economías distintas, implicará la convergencia en sus ingresos per cápita (aunque a distintas velocidades, por lo que se da el llamado principio de la dinámica de la transición).

Debe enfatizarse, por lo tanto, que dentro del modelo de Solow-Swan existe una convergencia de cada economía a su propio equilibrio de largo plazo. De allí que no es correcto exigir que se cumpla la convergencia absoluta, sino que se ha de encontrar una correlación parcial negativa entre crecimiento y nivel de ingreso, pero condicional al estado de equilibrio de largo plazo.

Otro conjunto de estudios señalan situaciones de disparidad a largo plazo. Esto es, apuntan a una divergencia final de posiciones. Más explícitamente, estas teorías no aceptan la existencia de un estado estacionario por la presencia de rendimientos decrecientes del capital; sostienen, en cambio, que el mecanismo ligado a la dinámica del capital no conoce ese límite. Hay fuerzas que operan evitando esa caída en el rendimiento del capital y, por lo tanto, niegan la presencia de una relación inversa entre el ingreso inicial y su incremento en el tiempo. Entre estas teorías están las conocidas como de crecimiento endógeno (autopotenciado), que se fundan sobre un conjunto de supuestos diferentes, pero se caracterizan por un elemento común: el abandono de la hipótesis de productividad marginal decreciente del capital que se asocia a la presencia de externalidades positivas y de escala. Esto es, si la productividad del capital no es decreciente, no hay razón teórica para sostener que las economías más pobres deban crecer más rápidamente que las más favorecidas.

Entonces, dichos modelos contrarios a la idea de convergencia sin calificativo (absoluta) se basan en la presencia de factores de crecimiento endógeno, como por ejemplo los que se sustentan en la existencia de rendimientos crecientes y economías externas espaciales (como los trabajos de Romer). Si los resultados empíricos avalan el modelo de Convergencia Beta absoluta, implica que todas las economías tienden a un mismo estado estacionario, y las divergencias presentes desaparecerán a largo plazo. En cambio, si el modelo válido es el de convergencia condicional, cada economía aparentemente tiende a un diferente estado estacionario. Lo cual, debe remarcarse, sin embargo, implica una estabilización en las diferencias de largo plazo.

Cabe apuntar que la cantidad de variables que poseen una alta correlación con el crecimiento 
en diversas regresiones ( $y$, por lo tanto, son consideradas determinantes del crecimiento) es enorme. Ello es un claro indicio de que la robustez de dichos resultados (o por lo menos de algunos) no es muy buena. En algunos trabajos, como los de Levine y Renelt (1992) y Temple (1995), se apunta a constatar la confiabilidad de los resultados obtenidos al utilizar diversos modelos y variables explicativas. El interés se centra, por lo tanto, en analizar la robustez de la significatividad estadística de los modelos ante cambios en la especificación. Así es que varios modelos parecen ser consistentes con la evidencia empírica, aunque lleven a conclusiones diferentes. Como consecuencia, no existe certeza sobre el verdadero modelo.

Ahora bien, todos los trabajos mencionados tocaban el problema con respecto a los territorios nacionales; pero las disparidades regionales, dentro de los países, constituyen un tema tanto o más preocupante, habida cuenta de que las diferencias regionales en la renta por persona (o en el nivel de bienestar) entre ciudadanos del mismo país resultan mucho menos excusables socialmente, justificables económicamente o aceptables políticamente. Sin embargo, en teoría, los procesos de aproximación son más factibles de observar entre regiones de un mismo país que entre naciones distintas, habida cuenta de que las similitudes deberán ser mayores en el primer caso. En especial, en cuanto hacen a los condicionantes institucionales.

Es decir, aun cuando los estudios de Convergencia Beta condicional o restringido se han utilizado ampliamente en casos subnacionales (es decir, convergencia interregional), ${ }^{2}$ desde el punto de vista teórico bien podría argumentarse que entre distritos o jurisdicciones del mismo país es esperable que opere la convergencia sin controles o condicionamientos (absoluta), considerando que deberíamos encontrarnos frente a economías muy similares, en virtud de su apertura económica mutua o integración, proximidades culturales,

2 Si se opera condicionando los datos con una serie de variables adicionales que marcan las diferencias relativas entre regiones ( $y$, por tanto, diferentes estados estacionarios). movilidad de los factores, funciones de producción semejantes, etc.

De allí que no tardaron en llegar los estudios regionales del caso. Entre los primeros, los más famosos, fueron los de Barro y Sala-i-Martin de 1990, 1991 y 1992, que estudiaron la convergencia entre Estados de la Unión Americana y 73 regiones de siete países europeos. Sus hallazgos apuntan a validar la tesis neoclásica de convergencia, señalando que las áreas inicialmente más desfavorecidas tienden a crecer más velozmente que las avanzadas, con un ritmo de convergencia cercano al $2 \%$ anual.

A la misma conclusión, es decir, presencia de convergencia entre áreas de un mismo país, llegan otros estudios referidos a distintos universos de investigación: Coulombe y Lee (1995) trabajaron sobre las provincias canadienses; Cárdenas y Ponton (1995), los departamentos colombianos; Mallick y Carayannis (1994), los estados mexicanos; Cashin (1995), el caso de los siete estados australianos; Chaterrii y Dewhurst (1996), los condados británicos.

En el caso de España, existen múltiples trabajos que arrojan resultados dispares. En Cuadrado Roura et al. (1999) se llega a la conclusión de que la convergencia entre las regiones españolas fue rápida durante los años cincuenta y sesenta, y se detuvo de modo brusco a finales de los setenta. Cuadrado Roura et al. (1999) sostienen que, en el caso español, la mayor parte del proceso de convergencia ocurrió por desplazamientos sectoriales y la consiguiente aproximación entre las estructuras productivas de las diferentes regiones (un cambio de estructura). Pero, tal vez, el proceso se haya ya agotado, y existan pocas posibilidades en el futuro de que esta convergencia continúe. A similares resultados, se llega en Cuadrado Roura et al. (2002). A su vez, en Cuadrado Roura y Maroto Sánchez (2010) se analiza la evolución regional en España del ingreso por habitante, pero especialmente la productividad y la estructura productiva, para el extenso periodo 1955-2006. Si se considera el conjunto del periodo, se puede decir que España registra una aceptable convergencia regional. Sin embargo, si se divide el lapso en subperiodos, aparecen claras diferencias: en el tramo 1955-1987, 
Alberto José Figueras • Daniela Cristina • Valeria Blanco • Iván Iturralde • Marcelo Luis Capello

el proceso de convergencia es claro en ingreso por habitante, con un valor beta de $-0,445$ y un $R$ cuadrado de 0,7765 ; mientras, en el subperiodo 1986-2006, la convergencia se redujo, con un beta de solamente $-0,1878$ y un escaso $R$ cuadrado de 0,3398 .

Los autores remiten este cambio en el proceso a circunstancias similares en variables como la evolución de la productividad y las estructuras productivas entre las diferentes comarcas. Realizan un análisis de Convergencia Sigma, y observan que en el medio siglo bajo consideración se da claramente, hacia el año 1980, un cambio de comportamiento. Se distingue un primer periodo (1955-1979) con una rápida convergencia en el ingreso por habitante (igual proceso se da en la productividad por ocupado, lo que reflejaría alteraciones en la composición sectorial del empleo, en un camino hacia una homogeneidad cada vez mayor). En cambio, el segundo subperiodo muestra una evolución diferente en la Convergencia Sigma: cesa durante los primeros años, para luego divergir y cerrar el registro con una ligera convergencia. El estudio señala hechos que explicarían estas circunstancias, como la evolución de la productividad y el empleo entre regiones. A su vez, se muestra una convergencia en el índice de especialización de las regiones (o comunidades autónomas) con un alto $\mathrm{R}$ cuadrado de 0,7589.

En una de las más recientes investigaciones (Garrido-Yserte y Mancha Navarro, 2010), se trabajó sobre Convergencia Sigma y Beta para el periodo 1986-2007. Los resultados de la Convergencia Sigma son apenas significativos; muestran un estancamiento en la dispersión de la variable producto regional per cápita, particularmente entre el 2002 y el 2007 (aunque se percibe convergencia dentro de clubes o grupos de regiones, similares en su producto per cápita). A similares conclusiones se arriba respecto de la Convergencia Beta. Estos hallazgos están en línea con investigaciones anteriores (Sotelsek y Marina, 2001), que señalaban un estancamiento de la convergencia en España (periodo 1979-1991). Pero pese a todo esto, las evidencias están muy lejos de ser concluyentes.
Otros estudios sobre las regiones del sur europeo, como en Tondl (1999) (estudio realizado para 38 regiones en España, Grecia e Italia meridional), también sugieren la importancia de factores como la educación y el capital social básico, factores en los que el proceso de catch-up bien puede haberse agotado. En definitiva, los resultados empíricos obligan a los autores a ser cautos en cuanto a la presencia de un verdadero y definitivo proceso de convergencia en Europa, en todos los países, incluso considerando idénticos periodos.

Las dificultades de aproximación entre las regiones están asociadas a la existencia previa de una mayor acumulación de capital, lo que genera habitualmente externalidades positivas. Sin duda que, pese a toda la idea de movilidad en la teoría, en el mediano plazo, los factores están localizados. Como solía afirmar Cuadrado Roura, no son objetivamente trasladables. Esto conduce a que los rendimientos del capital difícilmente sean decrecientes en las áreas geográficas donde la presencia de destacadas dotaciones de factores generan comparativamente más externalidades positivas, incluso pecuniarias.

Como se señala en Paci y Pigliaru (1997), en un trabajo sobre regiones italianas, los retornos a escala conducen a una causación acumulativa, lejana a la convergencia neoclásica (y más próxima a la visión de Myrdal-Kaldor). Llegamos, por lo tanto, a la perspectiva de divergencia, opuesta a la predicción neoclásica, y que inicia en los años cincuenta con Myrdal (1957) y se formaliza más tarde con Kaldor (1970).

Para el caso de las economías regionales, se destacan las investigaciones que señalan una mayor dispersión en el ingreso per cápita; al respecto, se encuentran los trabajos de López Bazo et al. (1997) para el caso de la Unión Europea, o de Mauro y Podrecca (1994), quienes examinan las regiones italianas, y apuntan a que lejos de converger, existiría un claro dualismo económico entre el norte y el mezzogiorno.

Tampoco se pueden olvidar los trabajos sobre convergencia y su posible vinculación con las transferencias interjurisdiccionales. Algunos trabajos 
encuentran efectos positivos en los niveles de transferencia en el caso de las provincias canadienses, como en Coulomb y Lee (1995) y Kaufman et al. (2003). En otras investigaciones, como Rodríguez (2006), por el contrario, no se encuentran efectos significativos. Para el caso de Australia, mientras Rangarajan y Srivastava (2004) hallan efectos positivos, en el estudio de Ramakrishnan y Cerisola (2004) no se encuentran efectos significativos. También, en otros países, lo hallado empíricamente es de lo más diverso: para Rusia, Martínez Vázquez y Timofeev (2010) obtienen efectos negativos; para la India, Bagchi (2003) llega a idéntica conclusión; pero para el caso de Brasil, De Oliveira (2008) y Maciel et al. (2008), arriban a la conclusión de que las transferencias han tenido su efecto positivo (aunque para un plazo más extendido, no hay evidencia de convergencia entre estados brasileños).

Hasta aquí, entonces, se puede decir que, en ciertos casos, como en la situación de los estados americanos, se ha dado una aproximación, de modo que los más rezagados han capturado, de alguna manera, a los más poderosos; pero, en otros casos, como entre las regiones italianas, no parece verificarse un acercamiento en sus performances económicas. Es de aclarar, no obstante, que pese a esa convergencia en Estados Unidos, que se apuntó líneas más arriba, aún hoy el grueso de su valor agregado nacional, como señala Krugman, se sigue concentrando en las proximidades de los Grandes Lagos y la costa atlántica noreste (a lo que deberíamos adicionar (alifornia), y el resto del producto bruto se distribuye en diferentes islas económicas dispersas.

En definitiva, hay una evidencia muy variable en cuanto a la convergencia. Esto se revela a través del peso que adquiere la variable dummy del Estado miembro, y también en los muchos estudios entre las regiones dentro de cada país. La polémica está muy distante de quedar zanjada, y es bastante posible que ambas situaciones coexistan en la realidad: convergencia y divergencia; en otras palabras, que determinadas zonas, en ciertas épocas y circunstancias, converjan, y en otros momentos, diverjan. ${ }^{3}$ Particularmente, en las últimas décadas, esto se conecta a las distintas capacidades de las diferentes áreas principales de las regiones periféricas y de los espacios en declive para adaptarse eficazmente a los desafíos vinculados a los procesos de cambio estructural que vienen exigiendo las nuevas tecnologías y la creciente liberalización. Incluso, para un contraste definitivo, no son ajenos los problemas de base de datos confiables. ${ }^{4}$

Para ser más explícitos, y resumiendo las proposiciones teóricas a partir del modelo de crecimiento de Solow, se presentan dos variantes: (a) el modelo básico (u original) de Solow, en el cual la tasa de crecimiento del ingreso por habitante de una región depende solamente del nivel de ingreso por habitante inicial del periodo bajo estudio, y (b) el modelo ampliado, condicionado por otros factores, que resulta ser una extensión del modelo básico. Aquí la tasa de crecimiento del ingreso per cápita no está solamente determinada por el nivel inicial, sino condicionada por otros determinantes (v. gr., el nivel de capital en diferentes variedades: humano, físico, infraestructura, etc.).

Estas variantes de modelización conducen a las siguientes predicciones: (a) el modelo básico original pronostica convergencia incondicional; (b) los modelos ampliados (o de crecimiento endógeno) pronostican convergencia condicional (en especial por el nivel de capital humano; es decir, cuando este es incorporado a través de otra variable explícita);

3 Como dato ilustrativo, en Cuadrado Roura et al. (2002) se señala que el impacto de la Unión Monetaria encerraba un gran riesgo, con inevitables ganadores y perdedores; siendo las áreas periféricas (sur de Europa e Irlanda), con bajo nivel de especialización, reducida flexibilidad en los mercados y bajo nivel de competitividad, quienes correrían mayor riesgo con la integración monetaria. Mientras que la zona central (Alemania, Holanda, Francia, Dinamarca) podría resultar favorecida en términos relativos, dadas sus características. La crisis del año 2008, con sus efectos hasta hoy, parece dar la razón a aquel pronóstico.

4 Debemos sumar a lo antedicho la perspectiva de divergencia ya mencionada, con ausencia de base neoclásica, aquella que comienza a defenderse, ya en los años cincuenta. Así, Myrdal (1957) plantea el concepto de causación acumulativa. Sus ideas fueron formalizadas por Kaldor (1970) y más tarde por Dixon y Thirwall (1975), con base en el concepto de rendimientos crecientes a escala en la industria manufacturera, manifestado en el coeficiente, o ley de Verdoorn (que refleja los efectos acumulativos del crecimiento a través de aumentos en la productividad). 
(c) lo anterior significa, en los hechos, una divergencia real, de acuerdo, por ejemplo, con el nivel de capital humano; de modo que los países que cuentan con más capital humano crecen más de prisa. Detengámonos, ahora, por un momento, en una breve revisión de los aspectos formales.

\section{Modelo de convergencia $\beta$ absoluta}

La convergencia $\beta$ absoluta se define como la existencia de una relación negativa entre el nivel alcanzado de producto per cápita y su tasa de crecimiento. Por ende, para medirla, se utiliza una ecuación de corte transversal:

$$
\ln \left(y_{t+T}^{i}\right)-\ln \left(y_{t}^{i}\right)=\theta+\gamma \ln \left(y_{t}^{i}\right)+u_{t}^{i}
$$

en la que $y_{t}^{i}$ representa el nivel de ingreso per cápita de la economía $i$ en el periodo $t$. Se debe verificar que el estimador del parámetro $\gamma$ sea negativo y estadísticamente significativo. La existencia de convergencia implica que un país (o región) pobre tiende a crecer más rápido que uno rico; de esta manera, dicho país (o región) tiende a alcanzar (catch-up) al rico en términos de ingreso per cápita.

\section{Modelo de convergencia $\beta$ condicional}

Siguiendo los conceptos de convergencia en el crecimiento (cfr. Barro y Sala-i-Martin, 1992), la tasa de crecimiento promedio del ingreso per cápita para la economía $i$ se puede estimar mediante la siguiente ecuación:

$\frac{1}{T}\left[\ln \left(y_{t+T}^{i}\right)-\ln \left(y_{t}^{i}\right)\right]=x+\frac{1-e^{-\beta T}}{T} \ln \left(y_{i}^{*}\right)-\left[\frac{1-e^{-\beta T}}{T}\right] \ln \left(y_{t}^{i}\right)+u_{t}^{i}$,

donde $x$ es la tasa de progreso tecnológico, $\mathrm{y}_{\mathrm{t}}^{*}$ es el nivel de ingreso per cápita de estado estacionario, $y u_{t}^{i}$ es el término de error. De esta manera, se usa el concepto de convergencia condicional ya que la tasa de crecimiento de la economía depende negativamente del nivel de ingreso inicial, después de que se condiciona por el estado estacionario.
Definiendo:

$x+\frac{1-\mathrm{e}^{-\beta T}}{T} \ln \left(y_{i}^{*}\right)=\theta,-\left[\frac{1-\mathrm{e}^{-\beta T}}{T}\right]=\gamma$, la ecuación a estimar es:

$$
\ln \left(y_{t+T}^{i}\right)-\ln \left(y_{t}^{i}\right)=\theta+\gamma \ln \left(y_{t}^{i}\right)+\delta X_{t}^{i}+u_{t}^{i}
$$

donde $X$ son variables condicionantes del crecimiento. La velocidad de convergencia se estima a partir de $\beta=-\frac{\ln (1+\gamma)}{T}$.

Por su parte, $\theta$ resume la información referente al cambio tecnológico y el estado estacionario. Cuando se tiene en cuenta que las economías comparadas poseen características estructurales diferentes, se deben incluir en el análisis los determinantes de esas diferencias en los estados estacionarios de las mencionadas economías. Este análisis permite indagar sobre qué variables son determinantes del crecimiento; de esta manera, se incluye en el análisis una cuestión tanto o más importante que el propio fenómeno de convergencia: los factores que permiten explicar las diferencias en las performances de largo plazo de las distintas economías.

\section{Convergencia Sigma}

El grado de diferencia entre las distintas área geográficas se mide por la varianza o el desvío estándar (DS) que existe en el grupo, tomando como referencia una determinada variable económica. Su evolución, o perspectiva dinámica, se ha denominado Convergencia Sigma. Cabe destacar, como aspecto teórico relevante que la evolución de sigma está también condicionada; esto es, depende de su valor de estado estacionario, para que exista convergencia, el DS debe bajar si es que el valor de sigma está por sobre su valor de estado estacionario, y deberá aumentar cuando su valor inicial sea menor al de largo plazo. Es decir, para remarcar, un DS decreciente solamente implica convergencia cuando esté por sobre su valor estacionario. 
Desde ya que es posible calcular el valor de DS en estado estacionario (Sala-i-Martin, 1999, cap. 10).

Aun cuando bajo convergencia se dé un descenso del valor de DS, el esquema teórico no concluye en que el valor del DS de estado estacionario sea cero, ya que depende de la varianza de los shocks aleatorios (último término de la expresión de cálculo). No puede cerrarse el asunto sin mencionar el cuestionamiento de Quah a los criterios de convergencia, particularmente a la Convergencia Beta, que llevó a la réplica de Sala-i-Martin (1999).

\section{EL CASO ARGENTINO: ESTUDIOS DE CONVERGENCIA ENTRE PROVINCIAS}

En cuanto al caso de Argentina, hay varios estudios sobre el problema del estancamiento y la posibilidad de convergencia en ingreso por habitante, desde los noventa, con Porto (1994, 1996), Willington (1998), Utrera y Koroch (1998), Marina (2001); y, luego, ya en este siglo, con Sotelsek y Marina (2001), Figueras, Arrufat y Regis (2003), Figueras, Arrufat, De la Mata y Álvarez (2004), Capello y Figueras (2006) o Capello et al. (2009). En estos trabajos se avanzó en lo atinente a las convergencias Beta y Sigma, así como en las variables que afectan los estados estacionarios (tabla 1).

En Willington (1998), se llega a la conclusión (con una base empírica) que las variables fundamentales que definen los distintos estados estacionarios para una convergencia condicionada resultan ser el nivel inicial de producto y el stock de capital humano preexistente. En Utrera y Koroch (1998), se apunta a que las cifras y la evidencia que han trabajado indican un efecto positivo sobre el nivel de los estados estacionarios tanto de la educación como de la inversión pública, y un efecto negativo del gasto público corriente.

En relación con la convergencia (o su contracara, la persistencia de las desigualdades entre provincias, e incluso de divergencia en su evolución), en Willington (1998) se señala terminantemente que cada provincia converge a su propio estado estacionario y a una velocidad diferente del resto. A su vez, la hipótesis de convergencia absoluta del Producto Bruto Geográfico (PBG) per cápita se rechaza en

Tabla 1.

Una síntesis de los principales aportes para Argentina

\begin{tabular}{|c|c|c|c|c|}
\hline Autor & Periodo analizado & $\begin{array}{c}\text { Convergencia Beta } \\
\text { absoluta }\end{array}$ & $\begin{array}{l}\text { Convergencia Beta } \\
\text { condicionada }\end{array}$ & Convergencia Sigma \\
\hline Porto (1994) & $1953-1980$ & Se rechaza & Se rechaza & \\
\hline Porto (1995 y 1996) & $1980-1988$ & No se rechaza & $\begin{array}{l}\text { No se rechaza } \\
\text { (condicionada por } \\
\text { infraestructura) }\end{array}$ & Se rechaza \\
\hline Utrera y Koroch (1998) & 1953-1994 & Se rechaza & No se rechaza & $\begin{array}{c}\text { No muestra tendencia } \\
\text { definida }\end{array}$ \\
\hline $\begin{array}{l}\text { Sotelsek Salem y } \\
\text { Marina (2001) }\end{array}$ & $\begin{array}{c}\text { Diferentes subperiodos } \\
\text { desde } 1954 \text { hasta } \\
1994\end{array}$ & $\begin{array}{c}\text { Se rechaza } \\
\text { (así para producto pc y } \\
\text { producto } \mathrm{p} / \text { trabajador) }\end{array}$ & $\begin{array}{c}\text { Se acepta para producto } \\
\text { pc } 1959 / 94 \text { y } 1983 / 94 \\
\text { Se acepta también para } \\
\text { producto por ocupado }\end{array}$ & \\
\hline $\begin{array}{l}\text { Russo y Ceña Delgado } \\
(2000)\end{array}$ & 1970-1995 & No se calcula & No se calcula & Se rechaza \\
\hline $\begin{array}{l}\text { Figueras, Arrufat y } \\
\text { Regis (2003) }\end{array}$ & $\begin{array}{l}\text { 1980-1998 (se trabaja } \\
\text { con series sin filtrar) }\end{array}$ & Se rechaza & No se rechaza & No se calcula \\
\hline $\begin{array}{c}\text { Figueras, Arrufat, De la } \\
\text { Mata y Álvarez (2004) }\end{array}$ & $\begin{array}{c}\text { 1980-1998 (se trabaja } \\
\text { con series filtradas) }\end{array}$ & Se rechaza & No se rechaza & $\begin{array}{c}\text { Se rechaza (DS estable, } \\
\text { con tendencia hacia la } \\
\text { divergencia }\end{array}$ \\
\hline
\end{tabular}

Fuente: elaboración de los autores. 
Utrera y Koroch (1998) y en Sotelsek Salem y Marina (2001); pero se acepta una convergencia condicional una vez que se mantiene constante el nivel de alfabetismo (Utrera \& Koroch, 1998). A similar conclusión llega Marina (2001), aunque con matices de acuerdo con los subperiodos de análisis (por ejemplo, se acepta una convergencia condicional para el periodo 1959-1994 y se rechaza para otros); se señala que los condicionantes (en especial, la alfabetización para el periodo 1959-1994 y 1983-1994) corrigen de modo definido la velocidad de convergencia.

En Utrera y Koroch (1998), se lee que el coeficiente beta de 0,03 (y significativo estadísticamente) con la condicionalidad del nivel de alfabetismo constituye evidencia en contra de los modelos de crecimiento endógeno y a favor del neoclásico, y, de acuerdo con los resultados, las provincias argentinas convergerían a diferentes estados estacionarios; lo que reduciría las brechas que las separan de estos a una tasa de aproximadamente el $3 \%$ anual y, como ya se mencionó, las diferencias en el nivel de educación afectarían en forma favorable el nivel de dichos estados estacionarios.

En Figueras et al. (2003), se trabajó la convergencia absoluta de las variables para el PBG y las nueve Grandes Divisiones (GD), y, a su vez, en tres periodos: el periodo total para el que se cuenta con datos (1980-998), y dos subperiodos (1980-1989 y 1989-1998). Para el caso de PBG per cápita, la evidencia en contra de la hipótesis de convergencia absoluta resulta ser muy fuerte. Atentos a esos resultados, los autores pasaron al análisis de la llamada convergencia condicional, analizando el crecimiento a partir de datos de panel, después de tener en cuenta explícitamente la diversidad de los estados estacionarios. Se concluyó que existía evidencia empírica que apoyaba fuertemente la existencia de Convergencia Beta condicional, con velocidades sustancialmente superiores al $2 \%$, lo que fue extensamente sugerido por los trabajos empíricos de los defensores de los modelos neoclásicos. A similares resultados llegaron Figueras et al., (2004), en esta oportunidad, a partir de series filtradas del efecto del ciclo.
Otro fenómeno a considerar, de la misma familia que la convergencia, es la aproximación entre economías regionales, en los valores de ciertas variables. Aunque ningún esquema teórico lo explique, por ahora, es un hecho que, en el caso concreto de las provincias argentinas, algunos indicadores, por ejemplo del mercado laboral (tasa de desempleo, tasa de ocupación, tasa de actividad), presentan una evolución que podríamos bautizar como equiparación (Figueras \& Ponce, 1998). En Porto (1994), se trabaja el mismo aspecto de convergencia en indicadores de bienestar corriendo una regresión simple por mínimos cuadrados ordinarios (MCO) entre tasa de crecimiento de cada índice durante el periodo y su nivel inicial; se observa una relación negativa ( $y$ significativa) en todos los casos (nivel de desarrollo, alfabetismo, necesidades básicas, mortalidad) entre las dos variables, lo que indica que se estaría ante un importante proceso de convergencia en bienestar (aunque esta circunstancia no se diera en el caso de producto por habitante). ${ }^{5}$ A similares conclusiones se llega en Figueras y Ponce (1998), si bien con base en distinto instrumental. Aspectos similares, en cuanto a convergencia en indicadores sociales, se han trabajado en Freille et al. (2011) y Capello et al. (2013).

Un análisis similar vinculado a los aspectos del mercado de trabajo se puede encontrar en el texto de Díaz Cafferata y Figueras (1999). Las conclusiones a que se llega allí es que, en alguna medida (al igual que en el caso de los indicadores de bienestar, o de desarrollo social) operaría una cierta convergencia, si se nos consiente el término.

No se puede dejar de enfatizar que el Estado argentino, consciente de estas diferencias espaciales

5 Se debe decir que la aproximación o convergencia en bienestar (aunque no necesariamente se logre en nivel de producto por habitante) es una meta anhelada. No se puede olvidar que el propósito de alcanzar una equidad horizontal (es decir, entre regiones) es, hoy por hoy, una ambición extendida en política económica. Así, en la última versión del Tratado de la Unión Europea, en Maastricht '92 y Ámsterdam '97, se enfatiza en que uno de los objetivos centrales es que los beneficios de la integración se distribuyan lo más ampliamente posible desde el punto de vista territorial, señalando acciones que permitirían lograr esa convergencia anhelada, y a ello apuntan los llamados fondos estructurales (Feder y FSE) y el Fondo de Cohesión. 
entre las provincias pobres y ricas, desde hace al menos tres décadas, ha operado un sistema de transferencias fiscales interjurisdiccionales fuertemente redistributivo, con propósitos equiparadores. Estas transferencias juegan, si se quiere, el mismo papel que los fondos estructurales en la Unión Europea (v. gr., el Fondo de Cohesión). Sin embargo, como se ha debatido en trabajos como Capello y Figueras (2006) y Capello et al. (2008), este flujo de recursos bien podría haber generado un efecto perverso, contrario a los propósitos, dando paso a una enfermedad holandesa fiscal entre provincias (Iturralde et al., 2012).

\section{ESTUDIO EMPÍRICO}

Para el conjunto del periodo bajo nuestro estudio, la economía del país ha transitado diferentes intentos de cambio en su estructura productiva. De un esquema propio del proceso de sustitución de importaciones, recomendado por la Cepal en los años sesenta, se pasó en los setenta y ochenta a un intento de apertura brusco entre 1976 y 1982, y pausado en la segunda mitad de los ochenta (con una economía argentina alejada del mercado mundial de capitales). Luego, en los noventa, la decisión aperturista fue definitiva, con una serie de medidas como el reingreso al mercado mundial de capital y el tipo de cambio fijo. Fracasada esta experiencia, con un derrumbe entre el 2001 y el 2002, se pasó a un esquema de crecimiento liderado por las exportaciones (dentro de lo que se suele denominar neomercantilismo), favorecido por el boom de los commodities y bajo un esquema de tipo de cambio flexible. Particularmente, este último esquema, finalmente similar en su "especialización" al modelo que insertará a Argentina en la economía mundial a finales del siglo XIX, tiene una gran trascendencia por resultar muy diferente de los distintos ensayos desde 1920.

La pregunta acerca de si se ha presentado convergencia, particularmente con datos del siglo $X X I$, tiene el atractivo adicional de obtener alguna información en cuanto a si este nuevo periodo de crecimiento nacional se ha distribuido en forma equitativa entre las regiones, contribuyendo a la igualdad o no.

En el acápite "Una perspectiva vigente: la convergencia económica" presentamos los aspectos formales de la convergencia absoluta y la convergencia condicionada. Ahora pasamos al estudio empírico. Nos centraremos en el modelo beta condicional. Reiterando lo ya dicho, este análisis permite indagar sobre qué variables son determinantes del crecimiento; esto es, los factores que permiten explicar las diferencias en las performances de largo plazo de las distintas economías.

Dada la falta de disponibilidad de información respecto del nivel de tecnología inicial, se plantea un tema no menor, pues, justamente, su tasa de variación resulta ser un factor principal en la explicación del crecimiento a largo plazo. Vale decir, en definitiva, que al ser una variable relevante en el análisis y que no puede ser incluida, implica la potencial existencia del sesgo de variable omitida, puesto que cuando las variables regresoras incluidas en el vector $X$ poseen algún tipo de correlación con una variable omitida, se produce un sesgo en los estimadores de los parámetros.

La utilización del modelo de datos de panel de efectos fijos permite captar su influencia: el nivel tecnológico inicial resulta una variable omitida que es constante en el tiempo, pero específica a cada una de las jurisdicciones $i$; por lo que su tratamiento como efecto fijo permite neutralizar el mencionado sesgo. De esta manera, se obtendría un $\theta_{i}$ para cada provincia que refleje la heterogeneidad en tecnología y estados estacionarios.

Dado que un conjunto de datos de panel consiste en observaciones de una muestra dada de individuos de una población, en el tiempo provee observaciones múltiples en el tiempo de cada individuo. Para el trabajo, se emplean series de tiempo para el periodo $1970-2007,6$ para las 23 provincias y la Ciudad Autónoma de Buenos Aires.

La variable dependiente es la tasa de crecimiento a diez años del PBG per cápita. Se utiliza la

$6 \quad$ La elección del periodo resulta de la disponibilidad de datos para un número significativo de provincias. 
definición de T=10 — cfr. ecuaciones (1) y (2)—, y se opta por emplear promedios de diez años para resumir la información de las variables a lo largo de estos años.

\section{Los condicionantes del estado estacionario}

En el modelo de Solow-Swan original, el estado estacionario está determinado ( $y$, por ende, la tasa de crecimiento en la transición) por los niveles de ahorro, la tasa de depreciación ${ }^{7}$ y la tasa de crecimiento poblacional. Dado que nuestra labor consiste en un análisis regional, es esperable desde la teoría encontrar convergencia sin necesidad de controlar variables, y como muestran los datos trabajados, en Argentina esto se cumple en un mínimo grado; por lo tanto, en una segunda etapa, se trabaja con variables que intentan reflejar las diferencias entre regiones. ${ }^{8}$ Antes de afrontar las pruebas econométricas, se ha reflexionado extensa y conceptualmente sobre las variables condicionantes.

No es menor el hecho de que debido a las limitaciones de información, no se pueda considerar entre los condicionantes una variable que, siguiendo la ley o regla de Thirlwall, sea crucial para explicar el desempeño de las provincias: sus ventas externas a la región (incluyendo sus exportaciones internacionales y sus ventas a otras provincias). Sería una mirada desde el export led growth model, considerando a las exportaciones como el componente más importante y autónomo

$7 \quad$ Bien mirado el cambio tecnológico que explica gran parte del crecimiento (esté o no explicado, dependiendo del modelo), este también tendrá, en los hechos, una arista negativa pues aumentará la depreciación real por la presencia de obsolescencia. Todo este juego de circunstancias nos hace pensar, aunque no podamos formalizarlo, que el cambio tecnológico generará discriminación entre áreas.

8 Ciertamente que el trabajar sobre múltiples regresiones, en un intento de hallar condicionantes, puede ser criticado; no obstante, entendemos que más que encontrarnos en la etapa epistémica denominada contexto de justificación de la teoría, estamos operando en el contexto de descubrimiento, haciendo algo así como un esfuerzo inductivo para encontrar factores diferenciales (de allí las innumerables pruebas econométricas). de la demanda agregada; ${ }^{9}$ variable que, en cierto modo, hemos trabajado en otras oportunidades de modo indirecto a través de las transferencias interjurisdiccionales (que pueden entenderse como flujos de capital que compensan los déficits o superávits de la balanza comercial de la provincia o la región). ${ }^{10}$

Sin duda, uno de los factores que distingue a las distintas provincias es el costo de transporte entre ellas y el gran núcleo de consumo del Litoral y principal área de embarque para el exterior, que funciona como centro en un vínculo centro-periferia. Este costo económico, o distancia virtual, se ha ido modificando con el paso del tiempo (y las distancias relativas se han alterado seguramente), ${ }^{11}$ lo que implica que, desde la teoría, hubiera sido una variable a incorporar, pero desdichadamente la carencia de ese dato crucial en una serie prolongada y para todas las jurisdicciones nos ha impedido incorporarlo (ya que la distancia geográfica pura presenta colinealidad con la constante de regresión).

Los términos de intercambio externos seguramente han jugado su papel en el último subperiodo, pero, al ser idénticos para todo el conjunto, no se lograría nada al incorporarlos explícitamente; pero su influencia se percibe en el cálculo de la Convergencia Sigma: las economías regionales del interior se han visto beneficiadas al hacer crecer sus ventajas competitivas. Para considerar su efecto, se ha incluido un índice de cambio estructural,

$9 \quad$ La ley de Thirlwall se inscribe en las explicaciones del estancamiento por restricción de demanda y no de oferta, sustentándose, finalmente, como el esquema centro-periferia de Prebisch, en una versión dinámica del multiplicador del comercio de Harrod. Sus ideas han sido cuestionada por Mc. Combie y Swales (1991) y Mc. Combie (1989), por supuestos demasiado restrictivos y una presunta falta de evidencia empírica suficiente. Sin embargo, en un entorno de desequilibrios interprovinciales, como en Argentina, y, aún con la debilidad de pruebas empíricas, parece brindar una explicación teórica aceptable y de sentido para los desequilibrios internos.

10 Las transferencias pueden ser vistas como compensadoras de déficits de balances regionales o como generadoras de esos déficits.

11 La variable distancia, o costo de la distancia, es el gran factor para la Nueva Geografía Económica, para gatillar tanto el proceso de concentración como el posterior de desconcentración. 
una variable construida teniendo en cuenta las diferentes estructuras sectoriales de las provincias.

Apuntado lo anterior, en cuanto a los determinantes del estado estacionario incluidos en el vector $X$, se consideraron las variables:

pbg_sumapbg: participación del PBG de cada provincia en el PBI del país;

gd5pc: GD 5, construcción (en miles de pesos de 1993) per cápita;

recopgtotal: cociente entre recursos tributarios propios de cada provincia y su gasto público;

gpersonal: cociente entre Gasto en Personal de cada provincia y su gasto público;

inversiongtotal: cociente entre gasto en inversión real directa de cada provincia y su gasto público total;

tasa_bruta: tasa bruta de mortalidad (como variable de salud);

gini: Coeficiente de Gini de distribución de ingresos en cada provincia;

esc_secundaria: se define como el ratio de alumnos matriculados en escuelas secundarias a la población total;

índice de cambio estructural, al que llama$\operatorname{mos} S_{i t}$. Se define de la siguiente manera:

$S_{i t}=\sum_{j=1}^{9} \omega_{i j, t-T} \cdot\left[\ln \left(\frac{y_{j, t}}{y_{j, t-T}}\right) / T\right]$, donde $\omega_{i j, t-T}$ es el peso del sector $j$ en la provincia $i$ en el momento $t-T$. Adviértase que $S_{i t}$ depende de las tasas de crecimiento de los sectores, y de los valores rezagados de participaciones sectoriales, lo que permite que esta sea tratada como exógena a la tasa de crecimiento actual de la provincia.

Para medir el capital humano, se pueden observar dos dimensiones: la educación, que mide el nivel y la calidad educativa, y la salud, donde se pueden emplear algunas medidas como la expectativa de vida o la tasa bruta de mortalidad. En el presente trabajo, se emplea la matrícula en escuelas secundarias en relación con la población total, como aproximación al nivel educativo, y la tasa bruta de mortalidad, como aproximación a la evolución a la situación de salubridad de la población.

La participación del PBG de la provincia con respecto al $\mathrm{PBI}$ nacional es considerada proxy de las economías de escala y externalidades positivas existentes en áreas de alta concentración económica. La participación de la GD5 (Construcción) en el PBG se emplea como aproximación de la tasa de inversión, aunque constituye una aplicación no reproductiva, y ello se justifica en que la construcción es el ítem más importante de la Inversión Bruta Interna Fija.

Al incluir la proporción de recursos tributarios propios con respecto al gasto público total de la provincia, se pueden identificar las provincias menos dependientes del gobierno nacional y con capacidad propia para llevar adelante políticas activas discrecionales. En aquellas provincias cuya proporción de sus fuentes genuinas de financiamiento en sus gastos totales sea mayor, es de esperar mayor capacidad de gestión y mayor eficiencia.

La proporción del Gasto en Personal en el Gasto Total de la provincia es un indicador de la importancia del empleo estatal en el mercado laboral, mientras que la proporción del gasto en inversiones en el Gasto Total intenta capturar la importancia de la inversión pública como motor de crecimiento.

La variable índice de cambio estructural mide el efecto de shocks exógenos sobre la tasa de crecimiento de cada región, y se incluye debido a que dichos shocks tienden a beneficiar o a perjudicar a provincias con altos o bajos ingresos (lo que haría que los shocks queden correlacionados con las variables explicativas), por lo que la omisión de $S_{i t}$ tendería a sesgar la estimación de los parámetros y la velocidad de convergencia (cfr. Barro \& Sala-iMartin, 2004, pp. 464-472). Esta variable revela a qué tasa crecería una provincia si cada uno de los sectores creciera a la tasa de crecimiento promedio nacional. Es decir, cuando se observa un valor bajo de la variable en una región, este indica que dicha provincia no crece rápido debido a un shock negativo en un sector relevante para su economía. Uno de los shocks que se espera que la variable capte es la influencia regional de las variaciones de los términos de intercambio.

En los modelos estándares, como se dijo, el ahorro es una variable clave. Lamentablemente, 
aunque se ha operado con diversas variables representativas bastante directas de la tasa de ahorro ( $v$. gr., el nivel de depósito regional en caja de ahorro y plazo fijo), estas variables no han respondido en su signo a los planteos teóricos. Dos alternativas caben: (a) el signo negativo responde a que se ahorra en la región, pero se aplica en otra región del espacio nacional, en virtud del nivel de concentración existente; (b) los depósitos no constituyen una variable suficientemente representativa del ahorro. Como consecuencia, se optó por una variable proxy más mediata: la distribución del ingreso (bajo ciertos supuestos generalmente utilizados).

El Coeficiente de Gini intenta captar la importancia potencial de la desigualdad en la distribución para la evolución comparada del nivel de ingreso. Un signo positivo implicaría un mayor nivel de acumulación en manos de las clases con mayor propensión a ahorrar, lo que conduce a un mayor nivel de crecimiento en la jurisdicción. Sin embargo, a su vez, la presencia de la desigualdad puede llevar a una inestabilidad social que, a largo plazo, puede resultar perjudicial para el proceso.

\section{RESULTADOS}

A continuación, y en los distintos acápites, presentamos los resultados obtenidos.

\section{Convergencia $\beta$ absoluta}

La tabla 2 presenta los resultados de la estimación del modelo de regresión lineal. Este se encuentra dividido en cuatro columnas. En la primera columna, se presentan los resultados para todo el periodo bajo análisis (1970-2007); mientras que los subperiodos analizados se visualizan en las siguientes columnas: década de 1980 (que analiza el crecimiento entre 1970 y 1989); década de 1990 (que analiza el crecimiento entre 1980 y 2001) y el periodo de 2000 (que analiza el crecimiento en el periodo 1992-2007).

Tabla 2.

Convergencia $\beta$ absoluta

\begin{tabular}{|c|c|c|c|c|}
\hline PBG & Todo el periodo & Periodo 1980-1989 & Periodo 1990-1999 & Periodo 2000-2007 \\
\hline Beta estimado & 0,00308 & $-0,00191$ & 0,00583 & 0,00261 \\
\hline logx0 $(\gamma)$ & $\begin{array}{c}-0,00303^{* * *} \\
(-4,77)\end{array}$ & $\begin{array}{c}0,00193 \\
(1,52)\end{array}$ & $\begin{array}{c}-0,00566^{* * *} \\
(-6,24)\end{array}$ & $\begin{array}{c}-0,00257^{*} \\
(-2,59)\end{array}$ \\
\hline Cons $(\theta)$ & $\begin{array}{c}0,0382^{* * *} \\
(5,58)\end{array}$ & $\begin{array}{c}-0,0127 \\
(-0,94)\end{array}$ & $\begin{array}{c}0,0661^{* * *} \\
(6,73)\end{array}$ & $\begin{array}{c}0,0314^{* *} \\
(2,89)\end{array}$ \\
\hline N & 670 & 240 & 288 & 142 \\
\hline Wald $\dagger$ & $\begin{array}{c}* * * \\
(22,71)\end{array}$ & $(2,31)$ & $(38,89)$ & $(6,69)$ \\
\hline
\end{tabular}

\begin{tabular}{|c|c|c|c|c|}
\hline PBG neto de transferencias & Todo el periodo & Periodo 1980-1989 & Periodo 1990-1999 & Periodo 2000-2007 \\
\hline Beta estimado & 0,00868 & $-0,00135$ & 0,01372 & 0,00707 \\
\hline $\log x 0 \_$nettransf $(\gamma)$ & $\begin{array}{c}-0,00832^{* * *} \\
(-5,23)\end{array}$ & $\begin{array}{c}0,00135 \\
(0,38)\end{array}$ & $\begin{array}{c}-0,0128^{* * *} \\
(-5,89)\end{array}$ & $\begin{array}{c}-0,00683^{* *} \\
(-2,94)\end{array}$ \\
\hline Cons $(\theta)$ & $\begin{array}{c}0,0105^{* * *} \\
(9,92)\end{array}$ & $\begin{array}{c}0,00768^{* * *} \\
(3,73)\end{array}$ & $\begin{array}{c}0,0119^{* * *} \\
(7,86)\end{array}$ & $\begin{array}{c}0,00876^{* * *} \\
(5,20)\end{array}$ \\
\hline $\mathrm{N}$ & 657 & 230 & 285 & 142 \\
\hline Wald $†$ & $\begin{array}{c}* * * \\
(27,30)\end{array}$ & $(0,15)$ & $\begin{array}{c}* * * \\
(34,65)\end{array}$ & $\begin{array}{c}* * \\
(8,63)\end{array}$ \\
\hline
\end{tabular}

Nota: estadísticos en $t$ paréntesis. $\dagger$ corresponde a una prueba $F{ }^{*} p<0,05,{ }^{* *} p<0,01,{ }^{* *} p<0,001$.

Fuente: elaboración de los autores. 
Los resultados muestran que para el periodo analizado, se encuentra una leve evidencia de convergencia absoluta, aunque con exasperante parsimonia: la demora sería de 225 años si consideramos el PBG y unos 80 años si operamos con PBG neto de transferencias (o sea, restadas las transferencias brutas recibidas por cada jurisdicción). En el subperiodo de 1980, tanto para el PBG total como para el PBG neto de transferencias, se rechaza la hipótesis de convergencia absoluta.

Sin embargo, cuando se analiza tanto el subperiodo de 1990 como el de 2000 , se encuentra evidencia empírica a favor del fenómeno de convergencia absoluta entre las provincias, ${ }^{12}$ siendo la velocidad de convergencia mayor cuando se trabaja con el PBG sin considerar las transferencias recibidas que cuando se considera el total del PBG (incluido el valor de las transferencias).

El análisis de la convergencia cuando se tiene en cuenta el PBG total, de manera comparada al PBG neto de las transferencias que reciben las provincias del gobierno nacional, merece un análisis especial. Se observa que, a pesar de que Argentina posee un sistema de transferencias

12 Es muy importante la variable dependiente a utilizar. Digamos, la variable a converger. Algunos autores llegan a la conclusión de que las convergencias mayores operan en las variables más cercanas al consumo (una explicación a esto sería que lo que converge es el bienestar más que la productividad). En nuestro caso, hemos realizado un análisis considerando los PBGpc y los PBGpc, sustrayendo las transferencias brutas, e intentando llevar el valor del ingreso a un valor más próximo al producto verdaderamente elaborado fronteras adentro de la provincia. Sin embargo, contrariamente a lo esperado, la velocidad de convergencia es mayor cuando se considera el PBG neto de transferencias (el producto) que cuando se considera el total del PBGpc (el ingreso). Quizá la explicación esté en que los valores no deberían restarse con transferencias brutas, sino con transferencias netas (o saldos fiscales netos), pero no contamos con una serie extendida de valores para tal cálculo. Otra explicación puede encontrarse en que los porcentajes de transferencias, al menos automáticas, a las distintas provincias, permanecen idénticos desde hace décadas, y al no variar en el tiempo, no afectan la Convergencia Beta (digamos que sería como un efecto fijo, inmodificable). No obstante, las transferencias colaboran en la disminución de la desigualdad año a año, lo cual se aprecia en los niveles de dispersión anual de los PBGpc provinciales (Convergencia Sigma): el DS, sin considerar transferencias (netas), es mayor que incorporando los valores transferidos brutos. En este sentido, las transferencias interjurisdiccionales colaboran en la nivelación (ver acápite correspondiente en este mismo trabajo). fiscales redistributivo entre provincias, la convergencia es mayor cuando se considera el PBG total que en el caso en que estas han sido detraídas del PBG. Esto sugiere que el sistema de transferencias no estaría ayudando a la convergencia, sino que, al contrario, estas estarían retardando el proceso de convergencia. Esta menor convergencia se puede deber al modo como se asignan dichas transferencias, lo que estaría en línea con un fenómeno tipo enfermedad holandesa en las áreas postergadas (cfr. Freille et al., 2011).

\section{Convergencia $\beta$ condicional}

Según se puede observar en la tabla 3, al permitir que la ordenada al origen de cada provincia varíe (esto es, la utilización de la especificación de efectos fijos, que permite distintos niveles de estados estacionarios y elimina el sesgo de estimación proveniente de la omisión del nivel tecnológico $\mathrm{u}$ otras variables determinantes), se aumenta de manera importante la velocidad de convergencia. En este caso, se trata de una convergencia condicional que resulta muy superior a la observada en la tabla 2, pues esta especificación ya considera el estado estacionario al que tiende per se cada una de las provincias.

A su vez, se observa que la evidencia a favor de la convergencia $\beta$ es clara (en todas las especificaciones se rechaza la hipótesis nula de $\gamma=0)$; de esta manera, se obtiene evidencia de un $\beta$ significativo y positivo (lo que, dada la expresión matemática utilizada, implica una relación inversa entre la tasa de crecimiento del PBGpc y su nivel inicial). Esto implica que cuando se realiza el análisis mediante datos de panel, con la especificación de efectos fijos, se concluye que hay evidencia empírica que sustenta la existencia de Convergencia Beta condicional, con velocidades de convergencia sustancialmente superiores al $2 \%$ (que es la cifra extensamente mencionada por los trabajos empíricos de los defensores de los modelos neoclásicos). Dada la velocidad de convergencia condicional obtenida, su tiempo sería de unos 20 años (según el cálculo a partir del PBG total). 
Tabla 3.

Convergencia $\beta$ condicional

\begin{tabular}{|c|c|c|c|c|}
\hline PBG & Todo el periodo & Periodo 1980-1989 & Periodo 1990-1999 & Periodo 2000-2007 \\
\hline Beta estimado & 0,03386 & 0,01637 & 0,05405 & - \\
\hline $\log x 0(\gamma)$ & $\begin{array}{c}-0,0287^{* * *} \\
(-21,48)\end{array}$ & $\begin{array}{c}-0,0151^{* * *} \\
(-3,60)\end{array}$ & $\begin{array}{c}-0,0418^{* * *} \\
(-22,59)\end{array}$ & $\begin{array}{c}0,00290 \\
(0,35)\end{array}$ \\
\hline Cons $(\theta)$ & $\begin{array}{c}0,314^{* * *} \\
(21,87)\end{array}$ & $\begin{array}{c}0,168^{* * *} \\
(3,77)\end{array}$ & $\begin{array}{c}0,456 * * * \\
(22,84)\end{array}$ & $\begin{array}{c}-0,0283 \\
(-0,31)\end{array}$ \\
\hline $\mathrm{N}$ & 670 & 240 & 264 & 142 \\
\hline Wald $†$ & $\begin{array}{c}* * * \\
(461,51)\end{array}$ & $\begin{array}{c}* * * \\
(12,93)\end{array}$ & $\begin{array}{c}* * * \\
(510,15)\end{array}$ & $\begin{array}{c}* * * \\
(0,12)\end{array}$ \\
\hline
\end{tabular}

\begin{tabular}{|c|c|c|c|c|}
\hline PBG neto de transferencias & Todo el periodo & Periodo 1980-1989 & Periodo 1990-1999 & Periodo 2000-2007 \\
\hline Beta estimado & 0,13209 & 0,08885 & $\ddagger$ & 0,03274 \\
\hline $\log x 0 \_$nettransf $(\gamma)$ & $\begin{array}{c}-0,0733^{* * *} \\
(-24,14)\end{array}$ & $\begin{array}{c}-0,0589 * * * \\
(-5,59)\end{array}$ & $\begin{array}{c}-0,100 * * * \\
(-27,06)\end{array}$ & $\begin{array}{c}-0,0279 \\
(-1,72)\end{array}$ \\
\hline Cons $(\theta)$ & $\begin{array}{c}0,0488^{* * *} \\
(26,84)\end{array}$ & $\begin{array}{c}0,0386^{* * *} \\
(7,11)\end{array}$ & $\begin{array}{c}0,0657^{* * *} \\
(28,60)\end{array}$ & $\begin{array}{c}0,0227^{*} \\
(2,12)\end{array}$ \\
\hline $\mathrm{N}$ & 657 & 230 & 261 & 142 \\
\hline Wald $†$ & $\begin{array}{c}* * * \\
(582,93)\end{array}$ & $\begin{array}{c}* * * \\
(31,23)\end{array}$ & $\begin{array}{c}* * * \\
(732,29)\end{array}$ & $\begin{array}{c}* * * \\
(2,96)\end{array}$ \\
\hline
\end{tabular}

Nota: estadísticos en $t$ paréntesis. $\dagger$ corresponde a una prueba F. $\bullet p<0,1 ;{ }^{*} p<0,05, * * p<0,01, * * * p<0,001$. $\neq$ logaritmo no definido; - no corresponde su cálculo.

Fuente: elaboración de los autores.

\section{Convergencia $\beta$ condicional y sus determinantes}

Una cuestión tanto o más importante que el propio fenómeno de convergencia es el análisis de los determinantes del crecimiento en el periodo estudiado; es decir, cuáles son las variables que, definiendo el estado estacionario, permiten explicar las diferencias en la performance de largo plazo de las economías provinciales. Vale decir que su análisis permite explicar a qué se deben las presumibles diferencias en las performances de largo plazo de las distintas economías provinciales. A continuación, se analizan las variables que resultan determinantes del estado estacionario en el periodo estudiado para las provincias argentinas (tabla 4).

La variable que mide la participación del PBG de cada provincia en el PBI del país (pbg_sumapbg) detenta un signo positivo, por lo que la concentración económica favorecería al crecimiento.

La proporción de recursos tributarios propios con respecto al gasto público total (recopgtotal) muestra un coeficiente positivo y estadísticamente significativo. Esto implica que aquellas provincias cuyos gobiernos provinciales poseen una capacidad de autofinanciación y una mayor independencia del gobierno nacional evidencian un mejor desempeño.

La proporción del Gasto en Personal en el Gasto Total de la provincia (gpersonal) ejerce un efecto negativo en las tasas de crecimiento. Es decir, a mayor gasto en empleo público en el mercado laboral, menor la tasa de crecimiento del producto.

La participación de la inversión en el Gasto Total (inversiongtotal) evidencia un efecto negativo en las estimaciones, por lo que, contrariamente a lo que se podría esperar, dicha variable tendría un 
Tabla 4.

Convergencia $\beta$ condicional (1970-2007)

\begin{tabular}{|c|c|c|c|}
\hline PBG & Efectos fijos & $\begin{array}{l}\text { Modelo de efectos fijos } \\
\text { con condicionantes }\end{array}$ & $\begin{array}{l}\text { Modelo con Driscoll -Kraay } \\
\text { standard errors }\end{array}$ \\
\hline Beta estimado & 0,03386 & 0,03502 & 0.03502 \\
\hline $\log x 0(\gamma)$ & $\begin{array}{c}-0,0287^{* * *} \\
(-21,48)\end{array}$ & $\begin{array}{c}-0,0295^{* * *} \\
(-5,35)\end{array}$ & $\begin{array}{c}-0,0295^{* * *} \\
(-5,35)\end{array}$ \\
\hline recopgtotal & & $\begin{array}{c}0,0115^{*} \\
(2,84)\end{array}$ & $\begin{array}{c}0,0115^{*} \\
(2,84)\end{array}$ \\
\hline gpersonalgtotal & & $\begin{array}{c}-0,0510^{* * *} \\
(-4,82)\end{array}$ & $\begin{array}{c}-0,0510^{* * *} \\
(-4,82)\end{array}$ \\
\hline Inversiongtotal & & $\begin{array}{c}-0,0294^{* *} \\
(-3,13)\end{array}$ & $\begin{array}{c}-0,0294^{* *} \\
(-3,13)\end{array}$ \\
\hline$g d 5 p c$ & & $\begin{array}{c}0,00000119 \\
(1,29)\end{array}$ & $\begin{array}{c}0,00000119 \\
(1,29)\end{array}$ \\
\hline Sit & & $\begin{array}{c}0,224^{* * *} \\
(6,31)\end{array}$ & $\begin{array}{c}0,224^{* * *} \\
(6,31)\end{array}$ \\
\hline pbg_sumapbg & & $\begin{array}{c}0,606^{* *} \\
(3,34)\end{array}$ & $\begin{array}{c}0,606^{* *} \\
(3,34)\end{array}$ \\
\hline esc_secundaria & & $\begin{array}{c}0,000379 \\
(0,99)\end{array}$ & $\begin{array}{c}0,000379 \\
(0,99)\end{array}$ \\
\hline tasa_bruta & & $\begin{array}{c}-0,00160^{* *} \\
(-3,78)\end{array}$ & $\begin{array}{c}-0,00160^{* *} \\
(-3,78)\end{array}$ \\
\hline $\operatorname{cons}(\theta)$ & $\begin{array}{c}0,314^{* * *} \\
(21,87)\end{array}$ & $\begin{array}{c}0,335^{* * *} \\
(16,86)\end{array}$ & $\begin{array}{c}0,335^{* * *} \\
(5,87)\end{array}$ \\
\hline $\mathrm{N}$ & 670 & 657 & 646 \\
\hline Wald & $\begin{array}{c}* * * \\
(461,51)\end{array}$ & $\begin{array}{c}* * * \\
(141,47)\end{array}$ & $\begin{array}{c}* * * \\
(607,82)\end{array}$ \\
\hline
\end{tabular}

Nota: estadísticos en paréntesis. ${ }^{*} p<0.05,{ }^{* *} p<0.01, * * * p<0.001$. El Test de Hausman arroja un $\chi^{2}(8)=259,66$, lo que implica al nivel de significatividad de 0,001 que se rechaza la hipótesis nula, por lo que el empleo de efectos fijos es adecuado. A su vez, la prueba $F(23,586)=52,65$ para testear la existencia de efectos fijos por año indica que a un nivel de significatividad de 0,001 , se rechaza la hipótesis nula (existen efectos fijos por año). A su vez, el Test de Pesaran de correlación contemporánea indica la presencia de dependencia cross-sectional. Finalmente, mediante el Test de Wald, modificado para groupwise heteroskedasticity, $\chi^{2}(24)=3601,68$, se rechaza la hipótesis nula de homoscedasticidad. Para corregir por heteroscedasticidad, autocorrelación y cross sectional dependence, se estiman errores estándar Driscoll and Kraay (cfr. Hoechle, 2007).

Fuente: elaboración de los autores.

efecto nocivo sobre el crecimiento, que podría estar vinculado a la ineficiencia del gasto gubernamental.

Sin embargo, la variable que se emplea como proxy de la propensión a invertir de cada provincia es el peso que presenta en el PBG la GD 5 , construcción (GD5pc). En tal sentido, el cálculo señala que influye positivamente en la tasa de crecimiento, por lo que, como es de esperarse, una mayor propensión a invertir sí redunda en un mayor crecimiento económico.
Las variables empleadas como proxy del capital humano merecen especial atención puesto que señalan que el capital humano es un factor que deriva en un mayor crecimiento económico. Esto se debe a que tanto un aumento en la escolarización secundaria (esc_secundaria) como una disminución en la tasa bruta de mortalidad (tasa_bruta) resultan en un mayor crecimiento económico.

A su vez, y como se señaló, una aproximación a los niveles de ahorro podría ser, de un modo 
mediato, el Coeficiente de Gini que presenta el signo positivo esperado (y significatividad). Por último, es palpable la importancia de los shocks estructurales en el proceso que estudiamos (es estadísticamente muy significativo), y además su coeficiente es muy elevado (aunque esto sería bastante relativo pues la variables no están normalizadas).

El coeficiente de la variable construida para captar los cambios estructurales muestra una fuerte y significativa influencia, lo que indica que la presencia de shocks exógenos altera sustancialmente la tasa de crecimiento de las provincias. Al mismo tiempo, dichos shocks tienen efectos diferenciados en las provincias según sea la estructura económica de su PBG. En la gráfica 1 se muestra la evolución del DS de dicha variable y se pueden observar, al menos, dos periodos en que la dispersión del efecto de los shocks entre provincias se agudiza; uno coincide con el periodo de apertura económica de los años noventa y otro con el boom de los precios de los commodities de la última década. Un análisis más profundo de los shocks captados por la variable índice de cambio estructural y su influencia en las economías regionales está en la agenda de futuras investigaciones.

Tabla 5.

Convergencia $\beta$ condicional (1972-2007) con Coeficiente de Gini

\begin{tabular}{|c|c|c|}
\hline PBG & Modelo de efectos fijos con condicionantes & Con Driscoll-Kraay standard errors \\
\hline Beta estimado & 0,03936 & 0,03936 \\
\hline $\log x 0(\gamma)$ & $\begin{array}{c}-0,0325^{* * *} \\
(-20,16)\end{array}$ & $\begin{array}{c}-0,0325^{* * *} \\
(-7,61)\end{array}$ \\
\hline recopgtotal & $\begin{array}{c}0,0111 \\
(1,78)\end{array}$ & $\begin{array}{c}-0,0325^{* * *} \\
(-7,61)\end{array}$ \\
\hline gpersonalgtotal & $\begin{array}{c}-0,0496^{* * *} \\
(-5,46)\end{array}$ & $\begin{array}{c}-0,0496 * * * \\
(-5,46)\end{array}$ \\
\hline Inversiongtotal & $\begin{array}{c}-0,0269 * * * \\
(-4,99)\end{array}$ & $\begin{array}{c}-0,0269 * * * \\
(-4,99)\end{array}$ \\
\hline gd5pc & $\begin{array}{c}0,00000218^{* * *} \\
(3,37)\end{array}$ & $\begin{array}{c}0,00000218^{* * *} \\
(3,37)\end{array}$ \\
\hline Sit & $\begin{array}{c}0,227 * * * \\
(11,34)\end{array}$ & $\begin{array}{c}0,227^{* * *} \\
(11,34)\end{array}$ \\
\hline pbg_sumapbg & $\begin{array}{c}0,549 * * * \\
(7,52)\end{array}$ & $\begin{array}{c}0,549 * * * \\
(3,91)\end{array}$ \\
\hline gini_provincial & $\begin{array}{c}0,0101 \\
(1,80)\end{array}$ & $\begin{array}{c}0,0101 \\
(1,25)\end{array}$ \\
\hline esc_secundaria & $\begin{array}{c}0,000409 \\
(1,38)\end{array}$ & $\begin{array}{c}-0,00181 * \\
(-2,53)\end{array}$ \\
\hline tasa_bruta & $\begin{array}{c}-0,00181 * * \\
(-2,95)\end{array}$ & $\begin{array}{c}0,000409 \\
(1,04)\end{array}$ \\
\hline $\operatorname{cons}(\theta)$ & $\begin{array}{c}0,365^{* * *} \\
(17,32)\end{array}$ & $\begin{array}{c}0,365^{* * *} \\
(8,17)\end{array}$ \\
\hline $\mathrm{N}$ & 575 & 575 \\
\hline Wald & $\begin{array}{c}* * * \\
(132,09)\end{array}$ & $\begin{array}{c}* * * \\
(310,17)\end{array}$ \\
\hline
\end{tabular}

Notas: estadísticos en paréntesis. ${ }^{*} p<0,05,{ }^{* *} p<0,01,{ }^{* * *} p<0,001$.

Fuente: elaboración de los autores. 
Para el subperiodo 1972-2007, se ha incluido asimismo el análisis con el Coeficiente de Gini (tabla 5). Según se observa, las estimaciones son consistentes con las que se presentan en la tabla 4. A su vez, el signo positivo que acompaña al estimador del Coeficiente de Gini implicaría que la mayor acumulación en manos de las clases con mayor propensión a ahorrar conduce a un mayor nivel de crecimiento.

\section{ESTUDIO DE CONVERGENCIA SIGMA}

Se puede comenzar con una referencia al concepto de convergencia nominal. La convergencia económica en su acepción nominal hace referencia, en general, a la homogeneización de las variables económicas nominales (González Sánchez, 2004). Por lo general, esta convergencia ha estado presente en la mira de autoridades como la Unión Europea, y ese criterio fue oportunamente tomado en el Tratado de Maastricht. En González Sánchez (2004), se referencia a De la Cámara Arilla, quien se refiere a la convergencia nominal como la "convergencia de las variables económicas más directamente responsables de la estabilidad de precios y tipos de cambio" (citado en González Sánchez, 2004, cap. III).

Es evidente que, implícitamente, en los procesos de integración, se considera a la convergencia nominal como una condición de la convergencia real. En el caso estudiado, dado que existe un mismo nivel de precios y un mismo nivel de tipo de cambio, los factores que deberían mostrar un proceso de aproximación nominal son las variables que definirían el estado estacionario. Intuitivamente, cuanto más homogéneos sean estos factores, más similares serán los estados estacionarios, y, por lo tanto, sería más posible la existencia de una efectiva aproximación real entre las economías.

En esta línea, puede ser muy indicativo la evolución del nivel de dispersión de las variables empleadas como control en la convergencia condicional (cuyos gráficos acompañamos), excepto las variables de escolaridad y el Coeficiente de Gini (cfr. nota metodológica).

Tabla 6.

Desvío estándar (DS) de las variables condicionantes

\begin{tabular}{|c|l|}
\hline Variable condicionante & \multicolumn{1}{c|}{ Comportamiento } \\
\hline Recursos propios sobre el PBG & Converge desde fines de los años ochenta. \\
\hline Gasto público en personal & $\begin{array}{l}\text { Aunque en los años setenta y ochenta se dio un proceso de aproximación, en los noventa } \\
\text { el DS se mantuvo estable. Desde finales de los noventa se da un proceso de convergencia. }\end{array}$ \\
\hline Gasto público en inversión & $\begin{array}{l}\text { Parece tener una tendencia bien divergente (con un gran pico de dispersión en el momento } \\
\text { de la crisis de la convertibilidad). }\end{array}$ \\
\hline $\begin{array}{c}\text { Gasto privado en inversión } \\
\text { (construcción) }\end{array}$ & $\begin{array}{l}\text { Esta variable, aunque inversión no reproductiva, resulta una proxy de la inversión a nivel } \\
\text { provincial (al no contar con cuentas provinciales por el método del gasto), y muestra un } \\
\text { proceso de convergencia. }\end{array}$ \\
\hline $\begin{array}{c}\text { Índice de cambio estructural } \\
\text { El peso del PBG de cada } \\
\text { provincia en el PBI }\end{array}$ & $\begin{array}{l}\text { Se percibe la influencia de los dos grandes shocks (la apertura y la crisis de México de } \\
\text { mediados de los noventa, y la crisis y salida de la convertibilidad, el aumento de los precios } \\
\text { de los commodities). }\end{array}$ \\
\hline $\begin{array}{l}\text { Se observa una fuerte caída en el DS, lo que se encuentra vinculado a la concentración } \\
\text { económica. }\end{array}$ \\
\hline $\begin{array}{l}\text { Índice de capital humano (salud) } \\
\text { nespués de un crecimiento de la desigualdad desde los años ochenta hasta finales de los } \\
\text { noventa, la evolución sugiere un proceso de convergencia en los años analizados del siglo XXI. }\end{array}$ \\
\hline
\end{tabular}

Fuente: elaboración de los autores. 
Gráfica 1 .

Sigma de las variables explicativas
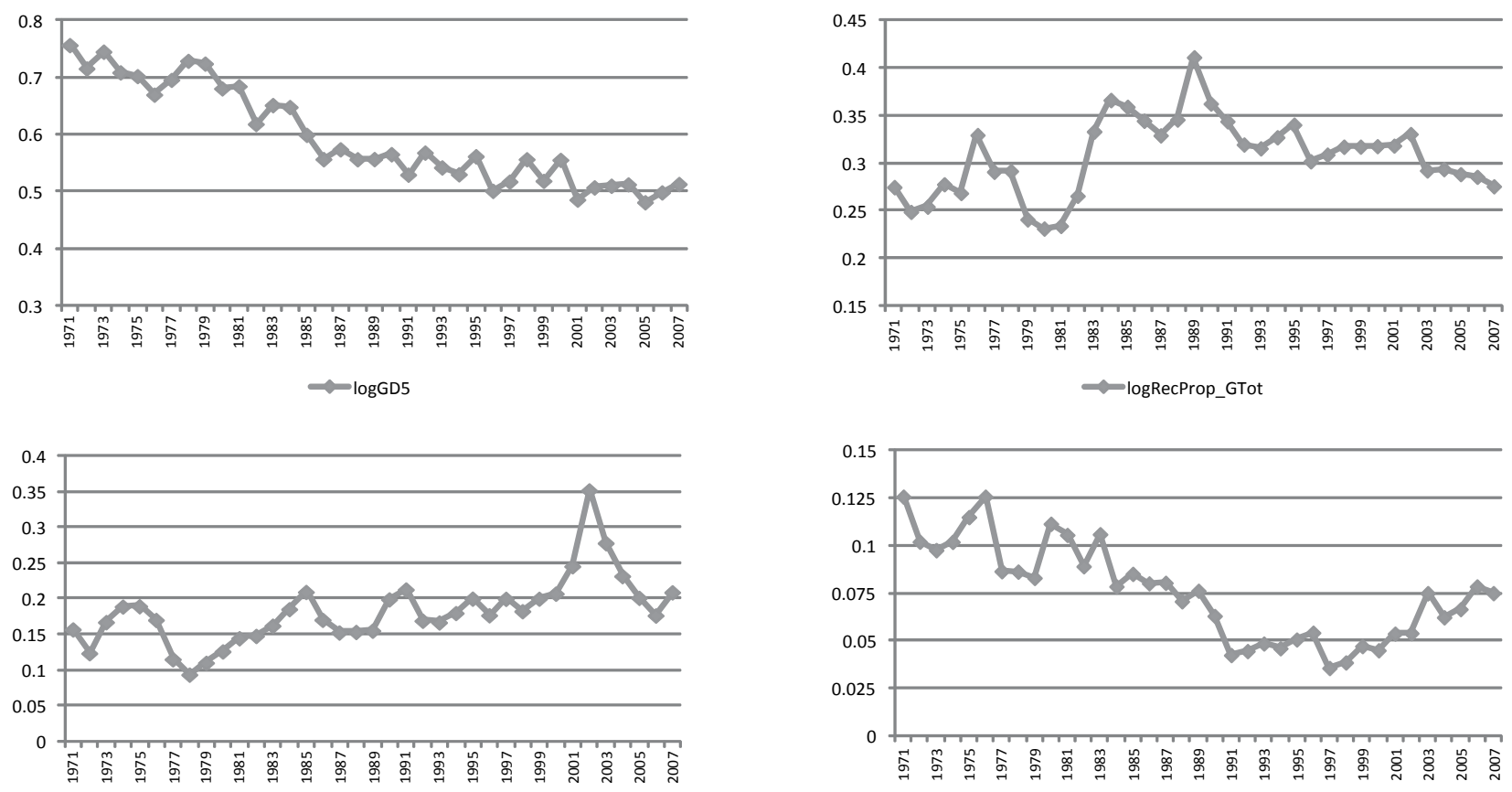

$\sim \log \operatorname{lnv} \_$GTot

$\sim \operatorname{logGPer}$ GTot

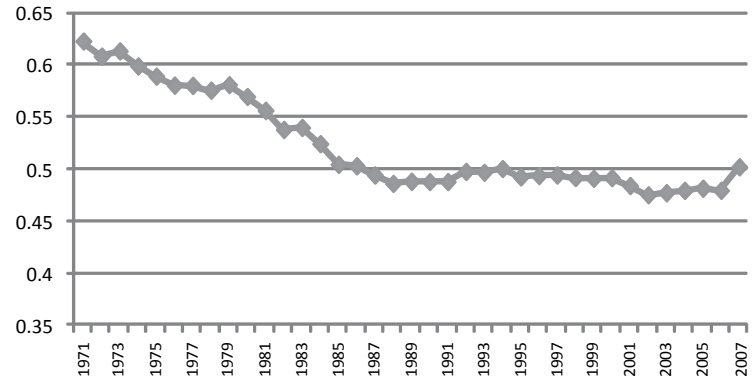

$\leadsto$ logPBG_sumaPBG
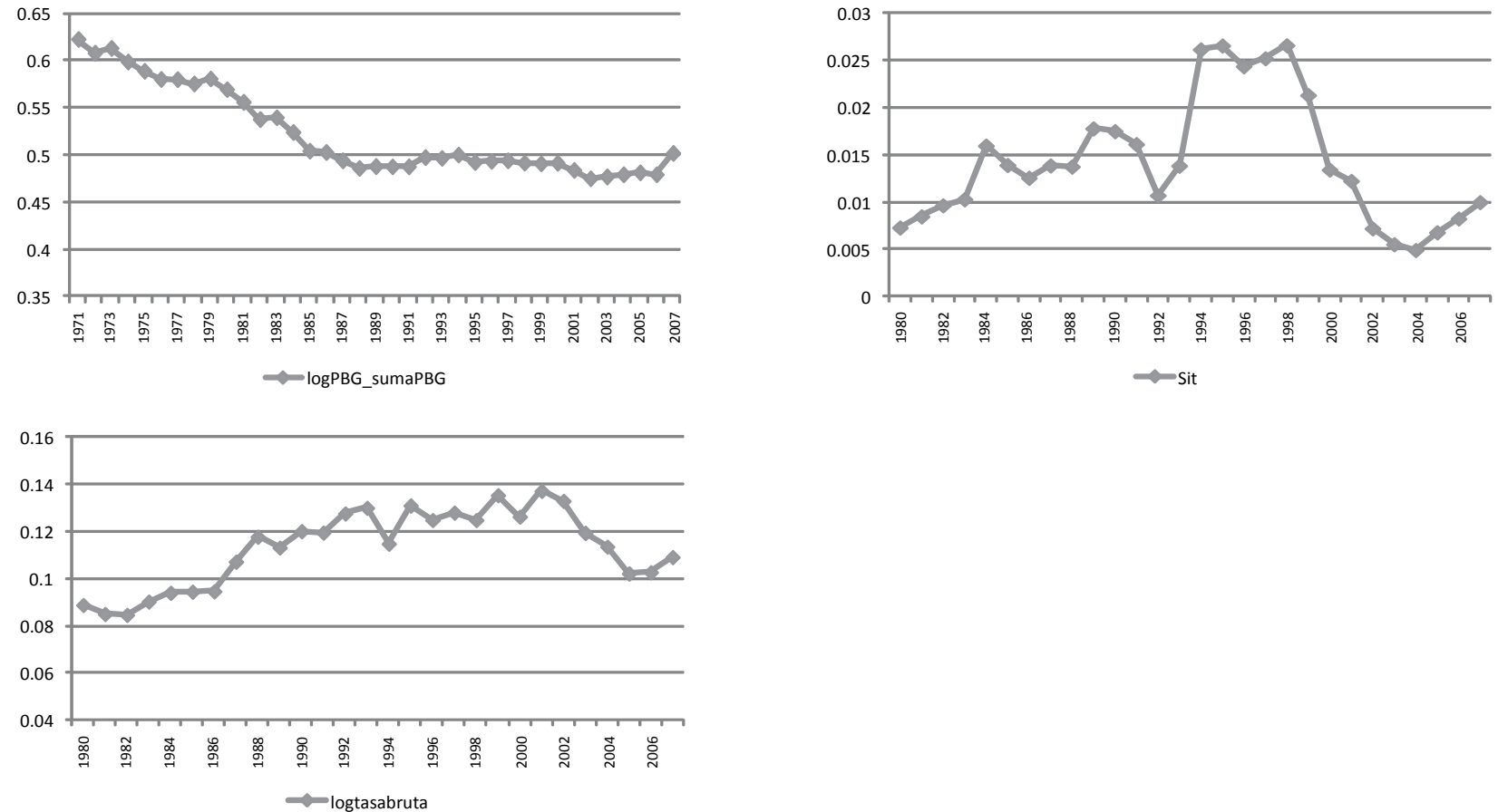

Fuente: elaboración de los autores. 


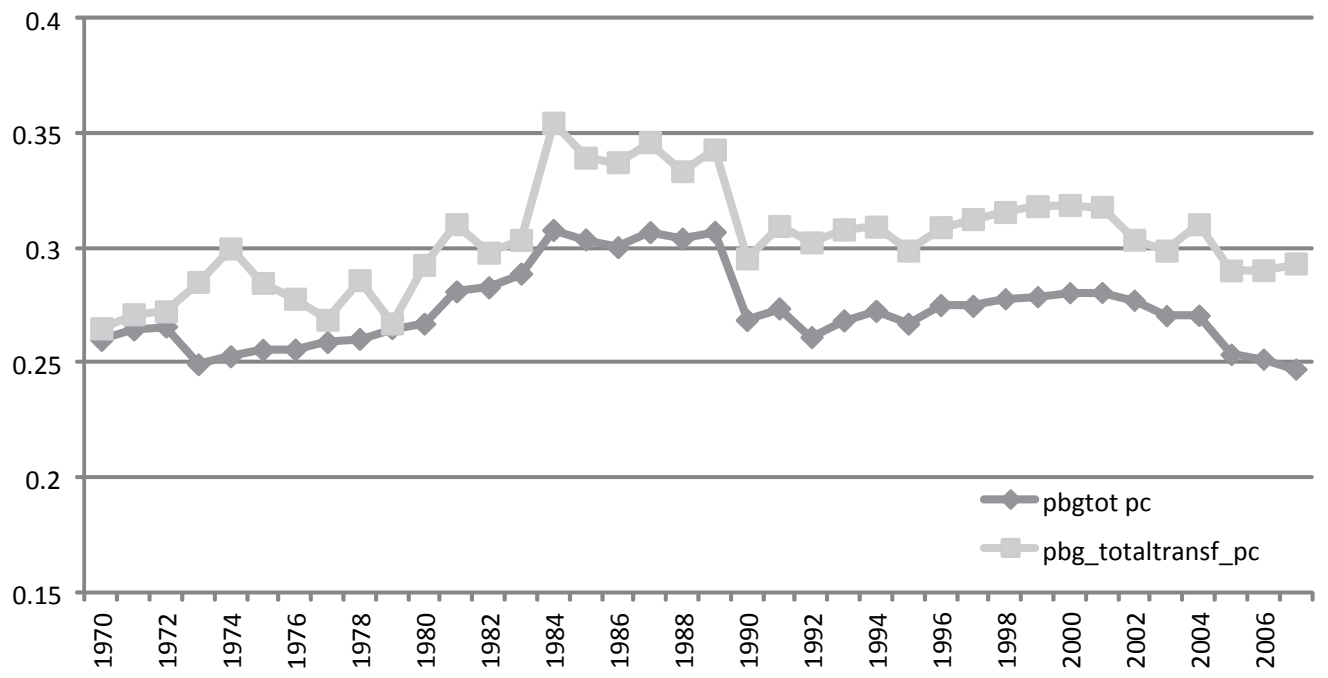

Nota: El $\sigma$ de estado estacionario se encuentra en todos los periodos por debajo del $\sigma$ observado. Fuente: elaboración de los autores.

Tabla 7.

Convergencia $\sigma(1970-2007)$

\begin{tabular}{|c|c|c|c|c|c|c|c|c|c|}
\hline & 1970 & 1975 & 1980 & 1985 & 1990 & 1995 & 2000 & 2003 & 2007 \\
\hline PBG & 0,26 & 0,26 & 0,27 & 0,30 & 0,27 & 0,27 & 0,28 & 0,27 & 0,25 \\
\hline PBGNetoTransf & 0,26 & 0,28 & 0,29 & 0,34 & 0,30 & 0,30 & 0,32 & 0,30 & 0,29 \\
\hline
\end{tabular}

Fuente: elaboración de los autores.

En la gráfica 2 se presenta la evolución del DS con dos líneas. La inferior contempla el cálculo a partir del producto tal como se brinda en las cuentas provinciales, mientras la línea superior presenta el DS del PBG una vez que se han restado las transferencias brutas recibidas. Es evidente que la presencia del sistema de transferencias, pese a sus deficiencias operativas, contribuye cada año a la equiparación de los ingresos per cápita ya que la línea que las contempla se encuentra por debajo de la línea del DS del PBG neteado de estas; lo que no implica que su efecto a largo plazo no sea dañino, como ya se mencionó en este texto (cfr. Freille et al., 2011).

Ahora bien, en ambas líneas se distinguen cuatro periodos: el primero, hasta 1983, de divergencia; una segunda etapa, que abarca los años ochenta de alto nivel de disparidad (hecho probablemente vinculado al proceso inflacionario; aunque no hay que descartar que ese mismo proceso haya generado ruido en los datos); un tercer periodo, que inicia en 1988/1989 con una brusca baja en el DS, manteniéndose estable en, aproximadamente, 0,27 (medida por PBG), y, finalmente, 
una cuarta etapa, que inicia hacia el año 2003, de descenso en los niveles de desigualdad regional. En este sentido, y respondiendo a la pregunta planteada anteriormente, se puede decir que la década de rápido crecimiento del siglo XXI, al menos hasta el año 2007, ha contribuido a equiparar las provincias en su PBGpc (muy posiblemente porque el crecimiento, gatillado por el precio de los commodities, se disparó inicialmente en el interior, donde se cuenta con ventajas comparativas).

En esta línea de estudio, también hemos analizado la performance de el DS por subgrupos. En primer lugar, se ha dividido el conjunto de jurisdicciones en tres grupos (si se quiere, clubes) de acuerdo con su peso en el producto nacional: las provincias más pequeñas en participación (sumadas las ocho, apenas llegan al 4,04\% del PBI de 1980 ), las provincias medianas (sumadas alcanzan el $8,32 \%$ del PBI) y las mayores (que aportan el $87,6 \%$ del PBI en los años ochenta).

En la gráfica 3 se muestra el DS del PBG per cápita según dos criterios de agrupación. El primero responde a la división según PBG (bajo, medio o alto) que tenían las provincias en el año 1980, mientras que el segundo responde a la agrupación según PBG per cápita (bajo, medio o alto) observado en las provincias en 1993.

Contrariamente a lo que suponíamos a priori, en el grupo de provincias más pequeñas (desde La Rioja, con el 0,20\% del PBI, hasta La Pampa, con el $0,83 \%)$ se da una convergencia en el ingreso por habitante. En el segundo grupo, el de las medianas, no existe una convergencia definida, aunque se percibe un descenso desde los años ochenta, cuando tuvo sus mayores valores de dispersión. Por último, entre las mayores, aquellas que mayor peso tienen en la estructura nacional, se percibe un proceso de divergencia, aunque leve.

Pero si clusterizamos por otra variable, concretamente el nivel de ingreso por habitante, llegamos a la conclusión de que hay divergencia entre aquellas provincias que en 1993 tenían menor nivel de vida. A su vez, las provincias de ingresos medios convergen fuertemente, y las de ingresos altos también lo hacen desde finales de los ochenta; por lo tanto, no se percibe, dentro de los grupos, en ningún caso, una tendencia clara durante los primeros años de este siglo.

A su vez, también hemos trabajado a partir de las regiones constituidas institucionalmente por propia iniciativa de las provincias durante las últimas décadas (gráfica 4). Estamos hablando en concreto de la Región Norte Grande, conformada en 1987 por Catamarca, Corrientes, Chaco, Formosa, Jujuy, Misiones, Santiago, Salta y Tucumán; la Región Nuevo Cuyo, de 1988, a la que pertenecen La Rioja, Mendoza, San Juan y San Luis); la Región Patagonia, de 1996, con Tierra del Fuego, Santa Cruz, Chubut, Río Negro, Neuquén y La Pampa, y, completando el mapa regional del interior, la Región Centro, de 1999, conformada por Córdoba, Santa Fe y Entre Ríos. Además, para cubrir todo el territorio nacional, creamos una región ad-hoc, en realidad inexistente en los papeles, con la Ciudad Autónoma de Buenos Aires (CABA) y provincia de Buenos Aires, que llamamos Región Porteña.

Al observar la evolución del DS, se percibe que los cuatro grupos constituidos formalmente convergen, considerando todo el periodo, aunque a ritmo desigual; mientras nuestra área construida ad-hoc (Región Porteña) diverge acentuadamente (gráfica 4 y tabla 8).

Por otra parte, cuando se analiza la evolución del DS entre regiones (y no intrarregiones), se observa que si se tiene en cuenta el interior y la Región Porteña, existe un proceso de divergencia. Pero si se analiza solamente el conjunto de las regiones del interior, no se percibe ninguna tendencia. Se mantienen, pues, las diferencias de partida existentes entre regiones hacia 1980.

Finalmente, en cuanto a los niveles de desigualdad entre regiones argentinas, y en una comparación internacional, se puede señalar que el nivel de desigualdad entre jurisdicciones en Argentina es superior al de Estados Unidos (Sala-i-Martin, 1999) y al de los países europeos (salvo Italia), al menos desde 1980 (Barro \& Sala-i-Martin, 2004). 
Sigma del logaritmo natural del Producto Bruto Geográfico (PBG) per cápita
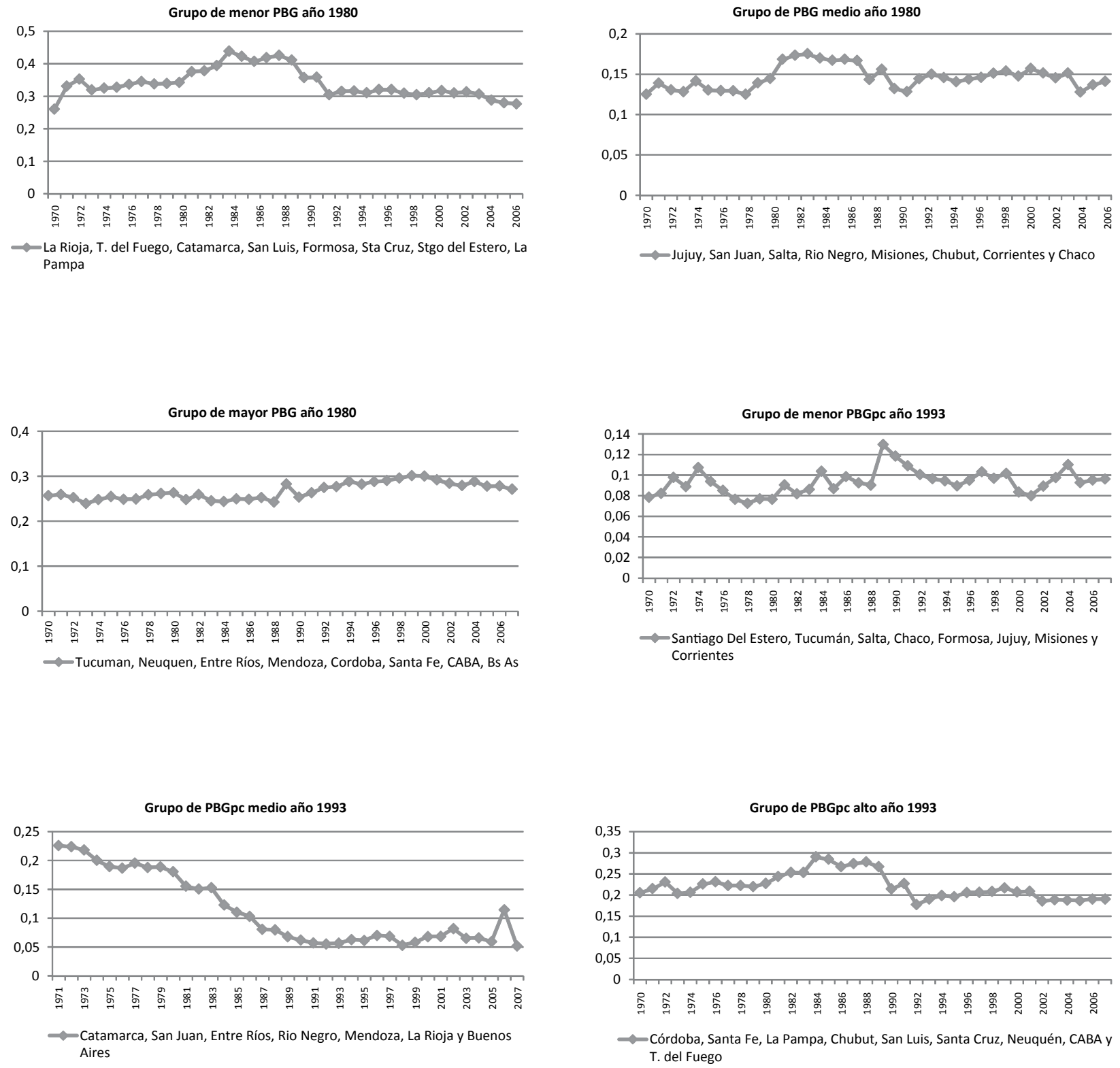

Fuente: elaboración de los autores. 
Gráfica 4 .

Sigma del logaritmo natural del Producto Bruto Geográfico (PBG) per cápita, agrupado según regiones
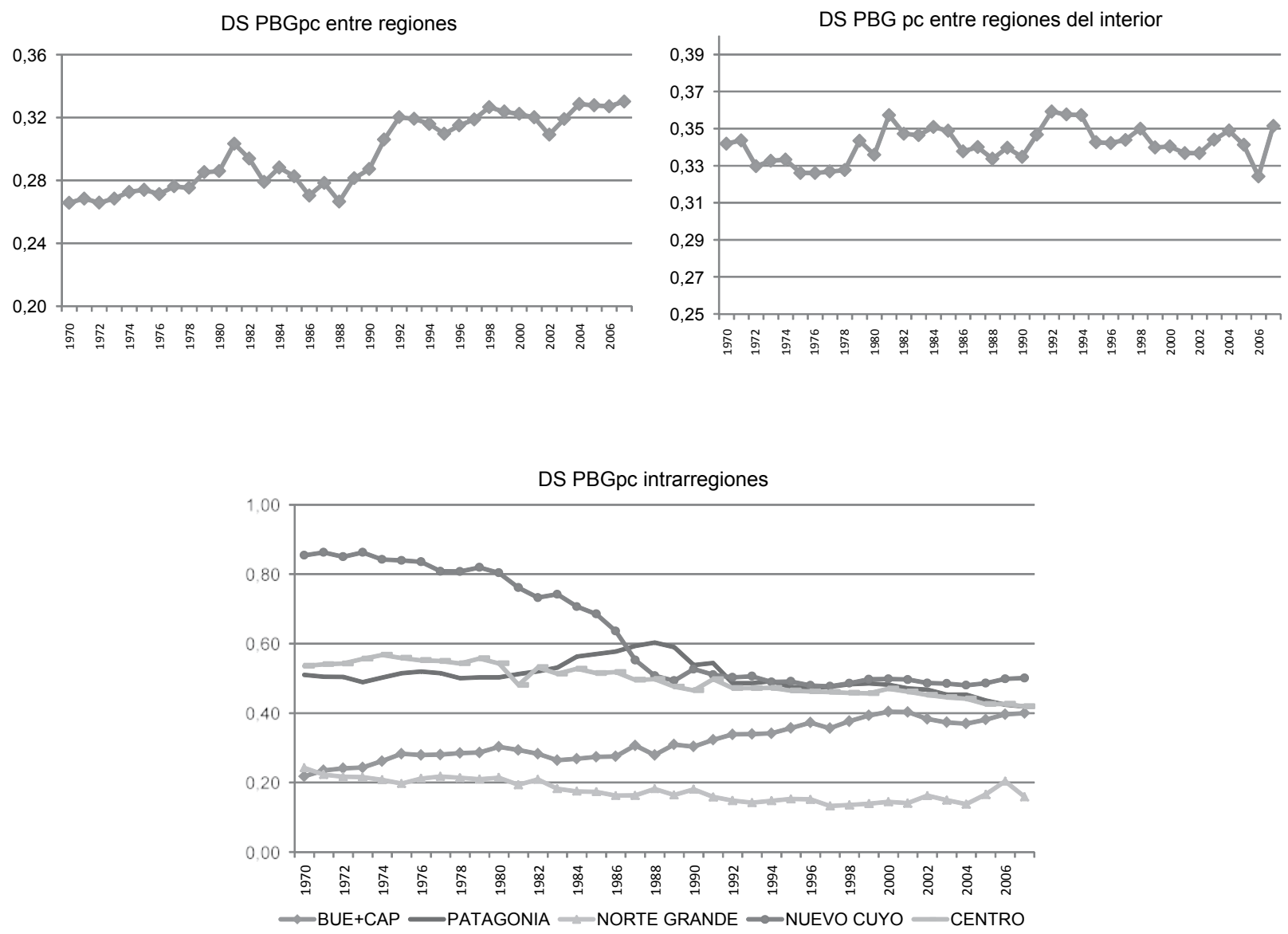

Fuente: elaboración de los autores.

Tabla 8.

Análisis de desvío estándar (DS) intrarregiones

\begin{tabular}{|c|c|c|c|c|c|}
\hline Región & Norte Grande & Nuevo Cuyo & Patagonia & Centro & $\begin{array}{c}\text { CABA + Bs. As. } \\
\text { (Región Porteña) }\end{array}$ \\
\hline Performance. & Converge. & $\begin{array}{c}\text { Converge } \\
\text { fuertemente. }\end{array}$ & $\begin{array}{c}\text { Converge desde } \\
\text { los noventa. }\end{array}$ & $\begin{array}{c}\text { Converge } \\
\text { levemente. }\end{array}$ & Diverge. \\
\hline
\end{tabular}

Fuente: elaboración de los autores. 


\section{CONCLUSIONES FINALES}

Los resultados del trabajo apuntan en dirección a una presencia de Convergencia Beta condicional. Acorde con la velocidad de convergencia obtenida, su tiempo sería de unos veinte años. A su vez, se puede decir que durante el episodio de crecimiento, ocurrido en el siglo que transcurre, la velocidad de convergencia al estado estacionario disminuye. Desde una perspectiva estática, la Convergencia Sigma indica una menor dispersión respecto de los años noventa.

Un punto importante en términos de implicancias de política surge de la comparación de los resultados de la convergencia cuando se tiene en cuenta el PBG total versus el PBG neteado de las transferencias que reciben las provincias del gobierno nacional. Este punto es relevante puesto que, aun cuando Argentina posee un sistema de transferencias fiscales fuertemente redistributivo entre provincias, precisamente la convergencia es mayor cuando se considera el PBG total que en el caso en que estas han sido detraídas del PBG. Esto sugiere que, contra la esperanza del sistema político, el esquema de transferencias estaría retardando el proceso de convergencia; hecho que puede deberse al modo en que se asignan dichas transferencias; hay evidencia de que se estaría generando en las áreas postergadas un fenómeno tipo enfermedad holandesa (cfr. Freille et al., 2011).

En otras palabras, a pesar de que por un efecto multiplicador favorable en el corto plazo estas transferencias favorecen el nivel de vida en las provincias, no necesariamente contribuyen al desarrollo cierto de sus fuerzas productivas (que se reflejaría en la evolución del producto por habitante). Se podría aseverar que quizá este subsidio espacial obstruya el mecanismo de convergencia al no incentivar la migración de los factores excedentes. De hecho, se encuentra evidencia de que aquellas jurisdicciones cuyos gobiernos provinciales poseen más capacidad de autofinanciación e independencia del gobierno nacional evidencian un mayor crecimiento. Esta situación está ligada, probablemente, a una mayor capacidad de gestión y eficiencia.
El análisis de los determinantes del crecimiento señala una incidencia positiva sobre el crecimiento, tanto de la propensión a invertir en la provincia como de la formación de capital humano en el área geográfica (en especial, en salud). Sin embargo, la inversión pública tendría un efecto adverso sobre el crecimiento; este es un punto que merecería un análisis ulterior más profundo.

Por otro lado, la mayor desigualdad en la distribución del ingreso muestra un efecto con signo positivo sobre el crecimiento. Este efecto, puede teorizarse, se explica por el hecho de que la desigualdad en la distribución genera mayor acumulación en manos de las clases con más alta propensión a ahorrar, con el consiguiente impacto sobre el crecimiento económico que habitualmente la teoría atribuye al ahorro. Sin embargo, no puede perderse de vista que, además de los aspectos éticos, la desigualdad puede atentar contra el mismo crecimiento económico ya que es fuente de inestabilidad social.

El presente trabajo aporta a la medición de los efectos de cambios estructurales sobre el crecimiento. Se observa que el desempeño de largo plazo de las economías provinciales se encuentra fuertemente condicionado por distintos shocks, que inciden de manera diferencial en las economías regionales. El coeficiente de la variable construida para captar los cambios estructurales $S_{i t}$ muestra una fuerte y significativa influencia, lo que indica que la presencia de shocks exógenos altera sustancialmente la tasa de crecimiento de las provincias. Al mismo tiempo, dichos shocks tienen efectos diferenciados en las provincias según sea la estructura económica de su PBG. A su vez, se observan al menos dos periodos en que la dispersión del efecto de los shocks entre provincias se agudiza, uno coincide con el periodo de apertura económica de los años noventa y otro con el boom de los precios de los commodities de la última década.

Otro condicionante es la participación del PBG de la provincia con respecto del PBI nacional. Su influencia positiva se puede deber al aprovechamiento de economías de escala en áreas de concentración económica. 
Por último, es necesario señalar que el trabajo presenta fuertes limitaciones, por razones vinculadas a las bases de datos (cfr. la nota metodológica). Pero la convergencia potencial, si existe, es un fenómeno de largo plazo, por lo cual la utilización de series temporales cortas no permitiría apreciar adecuadamente las tendencias; de allí que hayamos asumido el costo que implican los empalmes. Por otro lado, la convergencia/divergencia es el resultado de una serie de procesos de diferente naturaleza que no pueden ser captados en toda su magnitud por la simplificación propia de un modelo econométrico; especialmente si las fuentes de datos son intrínsecamente endebles (más allá del mencionado problema de los empalmes).

El análisis presentado, por otro lado, puede tener múltiples extensiones; una de ellas se refiere a estudiar la situación dinámica de largo plazo (por ejemplo, por series de Markov); otra, a trabajar no en base al ingreso por habitante (sea con transferencias o sin transferencias), sino por ocupado (en función de los ocupados estimados por provincia, lo cual estaría más cerca de la argumentación teórica, pero suponemos, por múltiples indicios, que su valor de convergencia será mucho menor); una tercera sería trabajar el proceso condicionado por efectos de contigüidad (v. gr., utilizando el Índice de Moran).

\section{NOTA METODOLÓGICA}

Las series de población y tasa de mortalidad se construyeron mediante una extrapolación lineal a partir de los valores obtenidos en los Censos de Población y Vivienda de los años 1970, 1980, 1991 y 2001.

Las series de PBG, a dos dígitos, periodo 19702007, se construyeron a partir de empalmes (por "retropolación") de series publicadas por el Consejo Federal de Inversiones (CFI) y J. Russo (1997). El Coeficiente de Gini fue obtenido de Cicowiez (2003), y Fossati (2002) (periodo 1984-2002), así como así también fue elaborado a partir de la Encuesta Permanente de Hogares (EPH) (periodo 2003-2007), donde los valores corresponden al dato de uno o más aglomerados (promediados) de cada provincia. Los datos de escolaridad secundaria se obtienen de los datos censales de Indec. Las series de depósitos y préstamos por división política provienen del Boletín Estadístico de Banco Central de la República Argentina para el periodo 1970-2009. 


\section{REFERENCIAS}

1. Adelman, I. (1974). Teorías del Desarrollo Económico. México: Fondo de Cultura Económica.

2. Ahumada, J. (1958). Problemas del desarrollo regional. Revista de Economía y Estadística, 3, 63-78.

3. Bagchi, A. (2003). Fifty years of fiscal federalism in India: An appraisal. Working Papers, 03(2), 1-76.

4. $\quad$ Bairoch, P. (1997).Victoires et déboires. Histoire économique et sociale du monde du XVIème siècle à nos jours. Paris: Gallimard.

5. Barro, R. J. (1991). Economic growth in a cross section of countries. Quarterly Journal of Economics, 106, 407-444.

6. Barro, R. \& Sala-i-Martin X. (1992). Convergence. Journal of Political Economy, 100(2), 223-251.

7. Barro, R. \& Sala-i-Martin X. (2004). Economic growth (2nd edition). Cambridge: MIT Press.

8. Baumol, W. (December, 1986). Productivity, growth, convergence and welfare: What the longrun data show. American Economic Review, 76, 1072-1085.

9. Baumol, W. J., Blackman, S. A. B. \& Wolff, E. N. (1989). Productivity and American leadership: The Long View. Cambridge: The MIT Press.

10. Canderlaria, C., Daly, M. \& Hale, G. (2009). Beyond Kuznets: persistent regional inequality in China. Working Papers Series, 7, 1-27.

11. Capello, M. \& Figueras, A. J. (2006). iLas transferencias fiscales producen enfermedad holandesa en las provincias? Documento presentado en Jornadas Internacionales de Finanzas Públicas. Córdoba, Argentina.

12. Capello, M., Figueras, A., Freille, S. \& Moncarz, P. (2009). Fiscal transfers, public sector wage premium and the effects on private wages. Revista de Economía y Estadística, 47, 41-66.

13. Capello, M., Figueras, A., Freille, S. \& Moncarz, P. (2013). The role of federal transfers in regional convergente in human development indicators in Argentina. Investigaciones Regionales, 27, 33-64.

14. Capello, M., Figueras, A., Grión, N. \& Moncarz, P. (2008). Los potenciales efectos perversos de las transferencias fiscales a los gobiernos subnacionales. Documento presentado en reunión AAEP. Córdoba, Argentina.

15. Cárdenas, M. \& Pontón, A. (1995). Growth and convergence in Colombia: 1950-1990. Journal of Development Economics, 47(1), 5-37.

16. Cashin, P. (1995). Economic growth and convergence across the seven colonies of Australia: 18611991. Economic Record, 71(213), 132-144.

17. Chatterji, M. \& Dewhurst, J. H. LL. (1996). Convergence clubs and relative economic performance in Great Britain: 1977-1991. Regional Studies, 30, 31-40.

18. Cicowiez, M. (2003). Caracterización económico-social de las provincias argentinas. Documento de Federalismo Fiscal, 5, 1-29.

19. Coulombe, S. \& Lee, F. (1995). Convergence across Canadian provinces, 1961 to 1991. Canadian Journal of Economics, 28(4a), 886-98.

20. Cuadrado Roura, J. R. (Ed.). (2010). Regional policy, economic growth and convergence. Heidelberg: Springer. 
21. Cuadrado Roura, J. R. \& Maroto Sánchez, M. (2010). Regional productivity convergente and changes in the productive structure. En J. R. Cuadrado Roura (Ed.), Regional policy, economic growth and convergente: Lessons from the Spanish case. Heidelberg: Springer.

22. Cuadrado Roura, J. R. \& Parellada, M. (Eds.). (2002). Regional convergence in the European Union. Heidelberg: Springer.

23. Cuadrado Roura, J. R., García-Greciano, B. \& Raymond, J. L. (1999). Regional convergente in productivity and productive systems: The Spanish case. Internacional Regional Science Review, 22, 35-53.

24. Cuadrado Roura, J. R., Mancha Navarro, T. \& Garrido Yserte, R. (1998). Convergencia regional en España: hechos, tendencias y perspectivas. Madrid: Fundación Argentaria.

25. Cuadrado Roura, J. R., Mancha Navarro, T. \& Garrido Yserte, R. (2000). Regional productivity patterns in Europe: An alternative approach. Annals of Regional Science, 34, 365-384.

26. Cuadrado Roura, J. R., Mancha Navarro, T. \& Garrido Yserte, R. (2002). Regional dynamics in the European Union: Winners and losers. En J. R. Cuadrado Roura \& M. Parellada (Eds), Regional convergence in the European Union. Heidelberg: Springer.

27. De Long, J. B. (1988). Productivity growth, convergence and welfare comment. American Economic Review, $78,1138-1154$.

28. De Oliveira, J. C. (Julho/Dezembro, 2008). Analise do crescimiento economico e das desigualdades regionais no Brasil. Estudos do CEPE, 28, 5-26.

29. Díaz Cafferata, A. \& Figueras, A. (1999). La desocupación en Argentina: una visión regional. Buenos Aires: Centro de Estudios Científicos y Técnicos-Federación Argentina de Consejos Profesionales de Ciencias Económicas (CECyT-FAPCE).

30. Dixon, R. y Thirlwall, A. P. (1975). A model of regional growth-rate differences on Kaldorian lines. Oxford Economic Papers, 27(2), 201-14.

31. Figueras, A. J. (2012a). Lecturas de economía argentina (y Regional). Córdoba: Eudecor.

32. Figueras, A. J. (2012b). El problema del crecimiento. En A. J. Figueras (Ed.), Lecturas de economía argentina (y Regional). Córdoba: Eudecor.

33. Figueras, A. \& Arrufat, J. (2009). El desafío del territorio. Córdoba: ACFCE.

34. Figueras, A. \& Ponce, C. (1998). El problema regional en Argentina. Análisis hasta los años 90. Serie de Estudios, IEF, UNC, 28, 1-74.

35. Figueras, A., Arrufat J. L. \& Regis, P. (noviembre, 2003). El fenómeno de la convergencia regional: una contribución. Documento presentado en reunión AAEP. Mendoza, Argentina.

36. Figueras, A., Arrufat, J., Blanco, V. \& De la Mata, D. (noviembre, 2005). Análisis de la movilidad regional en Argentina: un enfoque basado en las cadenas de Markov. Documento presentado en reunión AAEP. La Plata, Argentina.

37. Figueras, A., Arrufat, J., De la Mata, D. \& Álvarez, S. (noviembre, 2004). Convergencia regional: un estudio sobre indicadores de tendencia. Documento presentado en reunión AAEP. Buenos Aires, Argentina.

38. Foreman-Peck, J. (1998). Historia económica mundial. Relaciones económicas internacionales desde 1850. Madrid: P-Hall.

39. Fossati, V. (2002). Desigualdad y crecimiento. Un análisis para las provincias Argentinas (Tesis de maestría). Universidad Nacional de La Plata, Argentina. 
40. Freille, S., Figueras, A., Capello, M. \& Moncarz, P. (2011). Transferencias fiscales, el problema de la dádiva subnacional y la convergencia (económica y social). Documento presentado en Jornadas de Economía Aplicada. Málaga, España.

41. Friedman, M. (1992). Do old Fallacies ever die? Journal of Economic Literature, 30(4), 2129-32.

42. Fuentes, R. (1997). ¿Convergen las regiones en Chile? En F. Morandé \& R. Vergara (Eds.), Análisis empírico del crecimiento en Chile. Centro de Estudios Políticos, Santiago de Chile: Georgetown University.

43. Garrido Yserte, R. \& Mancha Navarro, T. (2010). The Spanish puzzle: Convergence, divergence and structural change. En J. R. Cuadrado Roura (Ed.), Regional policy, economic growth and convergence. Heidelberg: Springer.

44. González Sánchez, V. M. (2004). Convergencia económica en el Mercosur. Madrid: Ediciones UNED.

45. Hoechle, D. (2007). Robust standard errors for panel regressions with cross-sectional dependence. The Stata Journal, vv(ii), 1-31.

46. Iturralde, I., Figueras, A. \& Capello, M. (2012). El impacto de las transferencias: estudio comparado desde las transferencias brutas y desde los saldos fiscales netos a partir de nuevas evidencias. Documento presentado en Jornadas Internacionales de Finanzas Públicas. Córdoba, Argentina

47. Kaldor, N. (1970). The case of regional policy. Scottish Journal of Political Economy, 17, 337-348.

48. Kaldor, N. (1989). Capital accumulation and economic growth. En F. Targetti y A. Thirlwall, The essential Kaldor. London: Duckworth.

49. Kaufman, M., Swagel, P. \& Dunaway, S. (2003). Regional convergence and the role of federal transfers in Canada. IMF Working Papers WP/03/97, 1-13.

50. Levine, R. \& Renelt, D. (1992). A sensitivity analysis of cross-country growth regressions. American Economic Review, 82 (4), 942-963.

51. López Bazo, E., Vayá, E., Mora, A. \& Suriñach, J. (1997). Convergencia regional en la Unión Europea ante el nuevo entorno económico. Información Comercial Española, 762, 25-41.

52. Maciel, P. J., Andrade, J. \& Kuhl Teles, V. (2008). Tranferencias fiscais e convergencia regional no Brasil. En Working Papers. Brasil: Banco de Nordeste do Brasil.

53. Maddison, A. (1982). Phases of capitalist development. Oxford: Oxford University Press.

54. Mallick, R. y Carayannis, E. G. (1994). Regional economic convergence in Mexico: An analysis by industry. Growth and Change, XXV, 325-334.

55. Marina A. (2001). Convergencia económica en Argentina: ¿Qué nos dice la evidencia empírica?. En T. Mancha Navarro \& D. Sotelsek Salem (Eds.), Convergencia económica e integración. Madrid: Pirámide.

56. Martínez-Vázquez, J. \& Timofeev, A. (2010). Intra-regional equalization and growth in Russia. Working Papers, 10-11.

57. Mauro, L. \& Podrecca, E. (1994). The case of Italian regions: Convergence or dualism? Economic Notes, 23(3), 447-472.

58. Moreno, R. \& Vayá, E. (2002). Econometría espacial: nuevas técnicas, investigaciones regionales. España: Asociación Española de Centros Regionales, Universidad de Alcalá.

59. Myrdal, G. (1957). Economic theory and under-developed regions. Londres: G. Duckworth. 
60. Paci, R. y Pigliani, F. (1997). Structural change and convergence: An Italian regional perspective. Structural Change and Economic Dynamics, 8, 297-318.

61. Porto, G. (1994). Convergencia y política económica: algunos resultados para provincias argentinas (pp. 1191-1214). XXIX. Anales de la Reunión Anual de la Asociación Argentina de Economía Política, La Plata.

62. Porto, G. (1996). Las economías regionales en la Argentina. Buenos Aires: GEL.

63. Quah, D. (1993). Galton's fallacy and tests of the convergence hypothesis. Scandinavian Journal of Economics, 95(4), 427-443.

64. Quah, D. (1996). Regional convergence clusters accross Europe. European Economic Review, 951-958.

65. Ramakrishnan, U. \& Cerisola, M. (2004). Regional economic disparities in Australia. Working Paper, 04(144), 1-23.

66. Rangarajan, C. \& Srivastava, D. K. (2004). Fiscal transfers in Australia: Review and relevance to India. NIPFP Working Paper, 20, 1-60.

67. Ray, D. (1998). Development economics. Princeton: Princeton University Press.

68. Rey, S. J. \& Montouri, B. D. (1999). US regional income convergence: A spatial econometric perspective. Regional Studies, 33(2), 143-156.

69. Rodríguez, G. (2006). The role of the interprovincial transfers in the Beta: Further empirical evidence for Canada. Journal of Economic Studies, 33, 12-29.

70. Russo, J. (1997). Las disparidades regionales en Argentina y sus efectos sobre los sistemas agroalimentarios en el marco del Mercosur (Tesis doctoral). Departamento de Economía, Sociología y Políticas Agrarias. ETSIAM. Córdoba, España.

71. Russo, J. L. \& Ceña Delgado, F. (2000). Evolución de la convergencia y disparidades provinciales en Argentina. Revista de Estudios Regionales, 57, 157-173.

72. Sala-i-Martin, X. (1994). La riqueza de las regiones. Evidencias y teorías sobre crecimiento regional y convergencia. Moneda y Crédito, 198, 13-80.

73. Sala-i-Martin, X. (1999). Apuntes de crecimiento económico. Barcelona: Antoni Bosch.

74. Sotelsek Salem, D. y Marina, A. (2001). Convergencia en regiones españolas y provincias argentinas. En T. Mancha Navarro \& D. Sotelsek Salem (Eds.), Convergencia económica e integración. Madrid: Pirámide.

75. Targetti, F. \& Thirlwall, A. (1989). The essential Kaldor. Londres: Duckworth.

76. Temple, J. (1995). Testing the augmented solow model. Oxford: Nuffield College, Mimeo.

77. Thirlwall, A. P. (1978). La financiación del desarrollo económico. Barcelona: Macmillan-Vicens Vives.

78. Thirlwall, A. P. (2002).The nature of economic growth. Great Britain: Edward Elgar.

79. Tondl, G. (1999). What determined the uneven growth of Europe's southern regions: An empirical study with panel data, Research Institute for European Affairs. Working Paper, 30, 1-52.

80. Utrera G. \& Koroch, J. (1998). Convergencia: evidencia para las provincias argentinas. Documento presentado en la reunión AAEP. Mendoza, Argentina.

81. Willington, M. (1998). Un análisis empírico del crecimiento económico regional en Argentina. Estudios, 84, 13-36. 